\title{
Signaling mechanisms in cortical axon growth, guidance, and branching
}

\author{
Katherine Kalil ${ }^{1,2}{ }^{*}, L i L^{1,3}$ and B. Ian Hutchins ${ }^{1,4}$ \\ ${ }^{1}$ Neuroscience Training Program, University of Wisconsin-Madison, Madison, WI, USA \\ 2 Department of Neuroscience, University of Wisconsin-Madison, Madison, WI, USA \\ ${ }^{3}$ Pfizer, Inc., Groton, CT, USA \\ ${ }^{4}$ National institute of Neurological Disorders and Stroke, National Institutes of Health, Bethesda, MD, USA
}

Edited by:

Julian Budd, University of Sussex, UK

Reviewed by:

Carol Mason, Columbia University

Medical Center, USA

Sarah L. Pallas, Georgia State

University, USA

Susana Cohen-Cory, University of

California, USA

*Correspondence:

Katherine Kalil, Department of Neuroscience, University of

Wisconsin-Madison, 1300 University

Avenue, Madison, WI 53706, USA.

e-mail:kakali@facstaff.wisc.edu
Precise wiring of cortical circuits during development depends upon axon extension, guidance, and branching to appropriate targets. Motile growth cones at axon tips navigate through the nervous system by responding to molecular cues, which modulate signaling pathways within axonal growth cones. Intracellular calcium signaling has emerged as a major transducer of guidance cues but exactly how calcium signaling pathways modify the actin and microtubule cytoskeleton to evoke growth cone behaviors and axon branching is still mysterious. Axons must often pause their extension in tracts while their branches extend into targets. Some evidence suggests a competition between growth of axons and branches but the mechanisms are poorly understood. Since it is difficult to study growing axons deep within the mammalian brain, much of what we know about signaling pathways and cytoskeletal dynamics of growth cones comes from tissue culture studies, in many cases, of non-mammalian species. Consequently it is not well understood how guidance cues relevant to mammalian neural development in vivo signal to the growth cone cytoskeleton during axon outgrowth and guidance. In this review we describe our recent work in dissociated cultures of developing rodent sensorimotor cortex in the context of the current literature on molecular guidance cues, calcium signaling pathways, and cytoskeletal dynamics that regulate growth cone behaviors. A major challenge is to relate findings in tissue culture to mechanisms of cortical development in vivo. Toward this goal, we describe our recent work in cortical slices, which preserve the complex cellular and molecular environment of the mammalian brain but allow direct visualization of growth cone behaviors and calcium signaling. Findings from this work suggest that mechanisms regulating axon growth and guidance in dissociated culture neurons also underlie development of cortical connectivity in vivo.

Keywords: axon outgrowth, axon guidance, axon branching, calcium signaling, Wnt5a, CaMKII, corpus callosum, microtubules

\section{INTRODUCTION}

The development of appropriate connections is essential for the nervous system to function correctly. To achieve this motile growth cones at axon tips guide growing axons along appropriate pathways and into targets by responding to environmental guidance cues (Dickson, 2002). Thus intense interest has focused on the signaling mechanisms within the growth cone that link activation of surface guidance cue receptors to the actin and microtubule cytoskeleton that regulates growth cone motility and guidance behaviors (Dent and Gertler, 2003; Lowery and Van Vactor, 2009). It is well established, particularly in the mammalian central nervous system, that axons establish connections not only by activity of their growth cones but also by extending collateral branches from the shaft of the primary axon into the target (Kalil et al., 2000). This mechanism of interstitial branching (O'Leary et al., 1990 ) is especially important for the wiring of the cerebral cortex to distant targets in the spinal cord and contralateral cortex by efferent corticospinal and callosal axons. In cortical slice preparations direct visualization of cortical axons branching within the corpus callosum (Halloran and Kalil, 1994) and corticopontine tract (Bastmeyer and O'Leary, 1996) revealed that interstitial branching occurs from the axon shaft after the growth cone has extended past the target. Defining the signaling mechanisms that regulate axon growth, guidance, and branching is essential for understanding how the cerebral cortex becomes wired to appropriate targets during development. To address this question our laboratory used in vitro preparations of developing hamster sensorimotor cortex, which, in vivo, gives rise to the major efferent corticospinal and callosal pathways that contribute to motor, sensory, and cognitive functions. We identified guidance cues that promote cortical axon outgrowth, guidance, and branching, the intracellular calcium signaling pathways that evoke these axonal responses and the cytoskeletal mechanisms that regulate them. This review will focus on recent work from our laboratory and others showing how these cellular mechanisms shape the wiring of cortical connectivity during development. 


\section{GUIDANCE CUES PROMOTE OR INHIBIT CORTICAL AXON BRANCHING BY CYTOSKELETAL REORGANIZATION}

Cortical axons navigate over long distances by responses of their motile growth cones to attractive and inhibitory guidance cues in their environment. However, the growth cone at the tip of the primary axon does not typically grow directly into cortical and spinal targets. Instead branches arise interstitially from the axon shaft and then enter targets where they then form terminal arbors. Axon branching may be mechanistically linked to pausing behaviors of growth cones, which leave behind remnants on the axon at sites of future branching (Kalil et al., 2000; Dent et al., 2003). Growth cones consist of a central region which contains bundled microtubules and a thin expanded peripheral region dominated by actin filaments that form a meshwork in veil-like lamellipodia and bundles in fingerlike filopodia that protrude from highly motile lamellipodia (Figure 1). The growth cone responds to attractive cues by turning toward the source of the positive guidance cue whereas inhibitory guidance cues repel axons away from the repulsive guidance cue (Huber et al., 2003).

Guidance cues influence growth cone behaviors through cytoskeletal reorganization (Dent and Gertler, 2003; Kalil and Dent, 2005). Netrins, such as netrin-1, promote outgrowth of a wide variety of axons (Manitt and Kennedy, 2002) and attract growing cortical axons in explant cocultures (Metin et al., 1997; Richards et al., 1997). In contrast semaphorin 3A repels cortical axons in vitro (Bagnard et al., 1998; Polleux et al., 1998, 2000). However, little is known about effects of these attractive and repulsive guidance cues on cortical axon branching or the underlying cytoskeletal mechanisms. We therefore investigated effects of netrin-1 and semaphorin 3A on axon branching of cortical

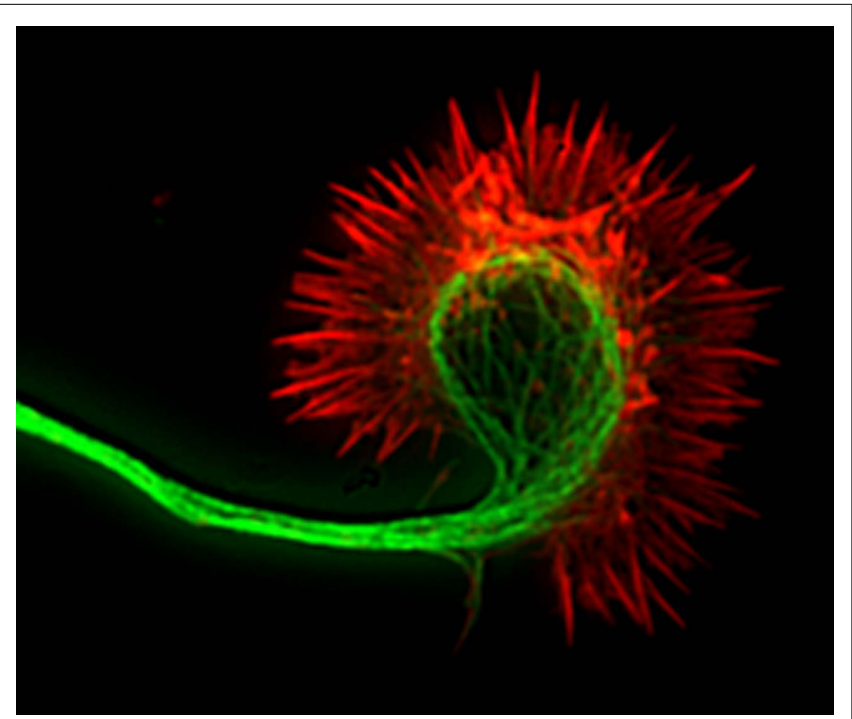

FIGURE 1 | Fluorescence image of a cortical axon and its growth cone. Actin filaments (indicated in red) are stained with phalloidin and occupy the lamellipodium and the spiky filopodia that protrude from its periphery. Microtubules (indicated in green) are stained with anti-tubulin antibodies and occupy the axon and central region of the growth cone. Dynamic microtubules can extend from the central region into the growth cone periphery to interact with actin filaments. neurons in dissociated culture (Dent et al., 2004) and found that netrin-1 increased branching by almost $60 \%$. Branches began as filopodia that grew to form stable branches within a few hours. Thus the effect of netrin-1 was to accelerate and increase branch formation. Local application of netrin-1 pulsed onto cortical axons evoked branching de novo from the axon shaft within minutes but had no effect on outgrowth of the primary axon, suggesting that axon outgrowth and branching are differentially regulated. Semaphorin $3 \mathrm{~A}$ had opposing effects, reducing axon branching and branch length without affecting axon length. Using live cell imaging of cortical neurons microinjected with fluorescent markers for actin filaments or microtubules, we investigated the cytoskeletal mechanisms underlying increased branching by netrin-1 or decreased branching by semaphorin $3 \mathrm{~A}$. In growth cones and at branch points along the axon shaft of cortical neurons interactions between dynamic microtubules (i.e., those able to grow and shrink in dynamic instability) and actin filaments are necessary for branch formation (Dent and Kalil, 2001). Netrin-1 increased actin filament bundles in the growth cone and axon shaft thereby increasing the number of filopodia along the axon shaft. Dynamic splayed microtubules interacted with the newly polymerized actin bundles to initiate new directions of growth by axon branches. In growth cones treated with semaphorin $3 \mathrm{~A}$ bundled actin filaments in the growth cone depolymerized, which reduced the motility and protrusion of filopodia and decreased microtubule exploration in the growth cone periphery. In collateral branches semaphorin 3A caused collapse of splayed microtubules and disappearance of actin bundles leading to a decrease in cortical axon branching. These results demonstrate that axon outgrowth and branching may in some cases be differentially regulated since netrin- 1 and semaphorin $3 \mathrm{~A}$ increased or decreased cortical axon branching respectively without affecting axon length. Moreover, development of axon branches involves changes at the growth cone as well as the axon shaft in cytoskeletal organization and dynamics. When actin/microtubule interactions are inhibited by guidance cues that depolymerize the cytoskeleton or inhibit their dynamics growth cones collapse and branching is inhibited.

\section{CALCIUM SIGNALING PATHWAYS MEDIATE NETRIN-1 INDUCED CORTICAL AXON BRANCHING}

The intracellular mechanisms that regulate axon branching independent of axon outgrowth are not well understood. Calcium is an essential second messenger that transduces axon guidance signals to regulate growth cone motility and pathfinding (Gomez and Zheng, 2006; Wen and Zheng, 2006; Zheng and Poo, 2007). In cortical neurons spontaneous global calcium $\left(\mathrm{Ca}^{2+}\right)$ fluctuations (transients) regulate axon outgrowth in a frequency dependent manner (Tang et al., 2003). Since netrin-1 induces extensive axon branching (Dent et al., 2004) and elevates $\mathrm{Ca}^{2+}$ levels in growth cones during steering events (Hong et al., 2000; Ming et al., 2002) we hypothesized that netrin-1 might promote axon branching through $\mathrm{Ca}^{2+}$ signaling events in the axon. Activation of specific signaling components is related to frequencies of $\mathrm{Ca}^{2+}$ transients. For example, calcium/calmodulin-dependent protein kinase II (CaMKII) functions as a spike-frequency detector (Hudmon and Schulman, 2002) and mitogen activated protein kinases (MAPKs) are also sensitive to intracellular $\mathrm{Ca}^{2+}$ changes 
(Cullen and Lockyer, 2002). Thus if repetitive $\mathrm{Ca}^{2+}$ transients are indeed involved in axon branching, CaMKII and MAPKs are likely candidates as downstream targets of $\mathrm{Ca}^{2+}$ signaling.

We investigated the role of calcium signaling in netrin-1 induced axon branching (Tang and Kalil, 2005) by loading cortical neurons with the membrane permeable calcium indicator dye Fluo-4AM, which detects changes in $\mathrm{Ca}^{2+}$ activity by changes in fluorescence intensity. Bath applied netrin-1 increased the average frequency of calcium transients fourfold either locally or globally in the axon. When netrin was withdrawn, $\mathrm{Ca}^{2+}$ activity declined to baseline levels. Reducing $\mathrm{Ca}^{2+}$ activity by blocking $\mathrm{IP}_{3}$ receptors on the endoplasmic reticulum to inhibit $\mathrm{Ca}^{2+}$ release from intracellular stores severely reduced branching. Local application of netrin-1 to the axon elicited large changes in $\mathrm{Ca}^{2+}$ in local regions of the axon. Remarkably, within minutes branches often protruded de novo from these regions of netrin-1 induced $\mathrm{Ca}^{2+}$ activity. Increased frequency of calcium transients by netrin-1 was strongly correlated with activation of CaMKII (as shown by increased phosphorylation). Whereas CaMKII inhibitors reduced cortical axon branching in the presence of netrin- 1 and also reduced axon outgrowth, over expression of $\alpha$ CaMKII increased axon branching. Conversely, RNAi knockdown of $\alpha$ CaMKII prevented netrin-1 induced axon branching. MAPK over expression increased axon branching whereas MAPK inhibition prevented netrin-1 induced branching but did not affect axon length. These results demonstrate for the first time that the axon guidance cue netrin-1 evokes rapid cortical axon branching through repetitive $\mathrm{Ca}^{2+}$ transients that activate the downstream kinases CaMKII and MAPK, which are sensitive to changes in the frequency of $\mathrm{Ca}^{2+}$ transients.

How are these findings relevant to cortical axon branching by netrin-1 in vivo? Netrin-1 is not expressed in the developing rodent cortical plate. Instead, the DCC ligand netrin-4 (Qin et al., 2007) is expressed in sensorimotor cortex at P6 (Takemoto et al., 2011) when mouse layer 2-3 axons are first extending branches into this region (Wang et al., 2007). Strong netrin-1 expression is observed in the cortical plate of the developing human fetus by 14 weeks of gestation (Harter et al., 2010), the time when callosal axons cross the midline and project to the contralateral cortex (Ren et al., 2006). This suggests that netrin-1 may have a larger role in human cortical development compared to rodents. In addition, DCC-expressing cortical axons extend collateral branches into the striatum (Sheth et al., 1998), which expresses netrin-1 at high levels throughout development (Livesey and Hunt, 1997; Takemoto et al., 2011). Taken together with our results on netrin-1 induced axon branching (Dent et al., 2004; Tang and Kalil, 2005), these data strongly suggest that during embryonic development netrins may direct the extension of collateral branches into the targets of cortical projection neurons.

\section{CALCIUM TRANSIENTS DIFFERENTIALLY REGULATE CORTICAL AXON OUTGROWTH AND BRANCHING}

In vivo and in vitro outgrowth of axons and the extension of branches are independently regulated such that branches can extend while their axons stall or retract (Luo and O'Leary, 2005). Thus, during the formation of corticospinal and callosal branches, axons have already completed their extension into efferent pathways (reviewed in Kalil et al., 2000). The mechanisms that regulate differential rates of outgrowth by axons and branches are poorly understood. One possibility, a competitive activity-dependent mechanism, regulates synaptic innervation at target sites. For example (Uesaka et al., 2006) in organotypic cocultures of thalamus and cortex neural activity enhanced the branch dynamics of thalamocortical axons that were biased toward more branch addition and elongation of branches in specific cortical target layers. The importance of electrical activity was further demonstrated in vivo where reducing neuronal activity in cortical neurons inhibited their morphological maturation including neurite elongation and branch development (Cancedda et al., 2007). Competition among neighboring axon arbors for synaptic space in the zebra fish visual system also favored arbors with higher levels of neural activity (Hua et al., 2005). Since electrical activity is reflected by changes in intracellular $\mathrm{Ca}^{2+}$ (Spitzer, 2006), we hypothesized that differences in levels of $\mathrm{Ca}^{2+}$ activity could also be a mechanism for regulating the differential growth of several processes of the same axon. This idea is supported by experiments with sympathetic neurons grown in divided chambers (Singh and Miller, 2005) which showed that one axon branch depolarized to increase $\mathrm{Ca}^{2+}$ activity grew longer at the expense of unstimulated branches in another chamber. This competition was dependent on calcium influx and downstream activation of CaMKII. However, these experiments could not distinguish between branches vs. axons from another cell and further investigation (Singh et al., 2008) suggested the involvement of neighboring axons.

To determine whether cortical neurons regulate axon outgrowth and branching by different levels of $\mathrm{Ca}^{2+}$ activity we measured spontaneous activity or manipulated calcium levels in an axon and its branches (Hutchins and Kalil, 2008). The frequencies of spontaneous $\mathrm{Ca}^{2+}$ transients were often different in a region of an axon vs. a branch or in one branch vs. another branch of the same axon (Figure 2). Higher frequency $\mathrm{Ca}^{2+}$ transients were correlated with more rapid outgrowth. Interestingly, differences in rates of outgrowth between processes of the same axon were dependent on relative differences in their $\mathrm{Ca}^{2+}$ transient frequencies. Processes with higher frequency transients were favored for growth while those with lower frequencies not only grew more slowly but retracted, suggesting a competitive effect. Moreover, the greatest differences in rates of outgrowth of two different processes of the same axon occurred when differences in frequencies were the highest. Global transients contributed relatively little to differential process outgrowth. To induce $\mathrm{Ca}^{2+}$ transients we applied BayK, which opens L-type voltage gated $\mathrm{Ca}^{2+}$ channels. BayK evoked localized calcium transients in cortical axons, which exhibited significant differences in outgrowth of processes belonging to the same axon. Over a 24-h time course both extension and retraction occurred in axons and their branches (Figure 3). $\mathrm{Ca}^{2+}$ imaging during the last $10-15$ min revealed that higher frequency $\mathrm{Ca}^{2+}$ transients were associated with increased rates of outgrowth vs. association of lower frequency $\mathrm{Ca}^{2+}$ transients with retraction or slower rates of outgrowth. We also used photolysis of caged calcium to evoke repetitive transients in restricted regions of axons and their branches (Hutchins and Kalil, 2008). This approach releases caged calcium in a region restricted to a small spot $(12-50 \mu \mathrm{m})$ and permits the choice of an optimal $\mathrm{Ca}^{2+}$ transient frequency by repetitive pulses of UV light. In most cases 

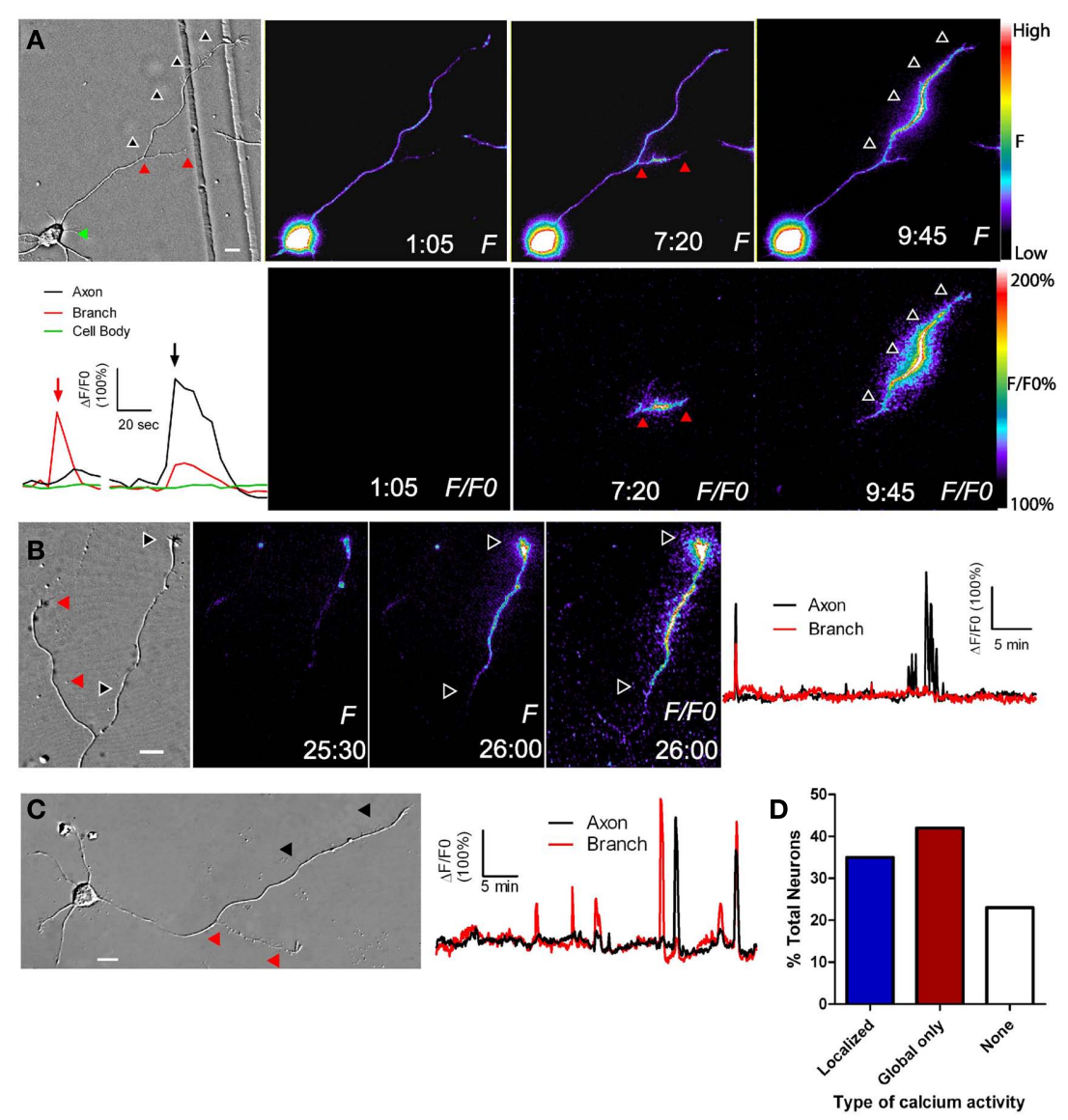

FIGURE 2 | Localized calcium transients occur in restricted regions of primary axons or their collateral branches. (A) DIC image at left and fluorescence images of calcium activity in a cortical neuron in which calcium transients are localized to the primary axon (at 9:45) or its collateral branch (7:20). At 1:05 there is no calcium activity. Black and red arrowheads correspond to regions of localized calcium activity. Panels F/FO show fluorescence change from baseline rather than raw fluorescence (lower panels). Pseudocolor calibration bars for images of raw fluorescence $(F)$ and fluorescence normalized to baseline (F/FO) are shown at right. The graph at left shows calcium activity in the primary axon (black), branch (red), and cell body (green) during calcium transients shown in the fluorescence images at
7:20 (red arrow) and 9:45 (black arrow). (B) DIC image (left) and fluorescence images of localized calcium activity indicated by black arrowheads in the primary axon of a cortical neuron (right panels). As shown in the graph of calcium activity (far right), localized calcium transients occurred only in the primary axon (black), and not in the branch (red). One global calcium transient occurred at the beginning of the imaging session. (C) Both the primary axon (black arrowheads) and branch (red arrowheads) show localized calcium transients but the branch has a higher frequency (graph at right). (D) Distribution of total cortical neurons with different types of calcium activity. Times are in minutes and seconds. Scale bar, $10 \mu \mathrm{m}$. (Reprinted from Hutchins and Kalil, 2008; with permission from the Society for Neuroscience). the stimulated process was favored for growth while unstimulated processes often retracted. Thus results from spontaneous and induced $\mathrm{Ca}^{2+}$ activity revealed similar competitive effects on the growth of one axonal process at the expense of another by differential frequencies of $\mathrm{Ca}^{2+}$ transients.

How do results from these in vitro experiments shed light on mechanisms of cortical axon branching in vivo? Cortical axons in pathways such as the corpus callosum vs. their branches projecting into cortical target regions could encounter different guidance cues such as netrin-1 at varying concentrations that evoke different levels of $\mathrm{Ca}^{2+}$ activity. This could lead to competition among different processes of the same axon for growth such that the primary axon could stall or retract while a branch of the same axon could extend into targets. In living cortical slices (Halloran and Kalil, 1994), we observed these events directly in the developing corpus callosum. Different frequencies of $\mathrm{Ca}^{2+}$ transients could regulate the differential outgrowth of an axon vs. its branch by activation of calcium frequency dependent effectors such as CaMKII and MAPK in each axonal process. Effectors such as CaMKI and CaMKII promote neurite outgrowth (Borodinsky et al., 2003; Wayman et al., 2004, 2006) and CaMKII is preferentially activated in cortical axon branches with higher frequency $\mathrm{Ca}^{2+}$ transients (Tang and Kalil, 2005). CaMKII $\beta$ binds to actin filaments and increases filopodial dynamics (Fink et al., 2003), consistent with the observation that direct elevation of $\mathrm{Ca}^{2+}$ activity by photolysis of caged calcium can rapidly evoke protrusion of new axonal filopodia by actin filament polymerization (Lau et al., 1999). Calcium transients of different frequencies may target different kinases and phosphatases such as CaMKII and calcineurin in the cytoplasm to evoke different cellular responses (Tomida et al., 2003). Events, such as extension and 

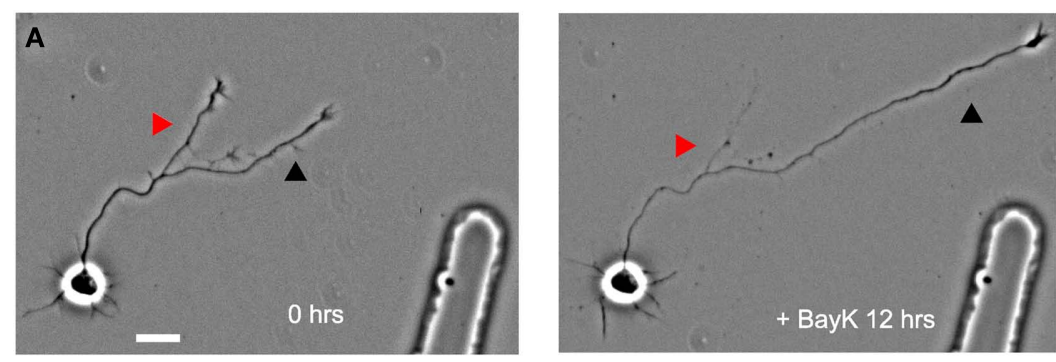

B

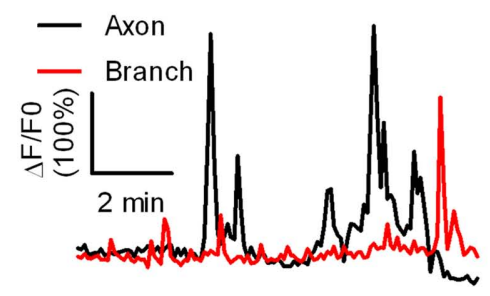

C

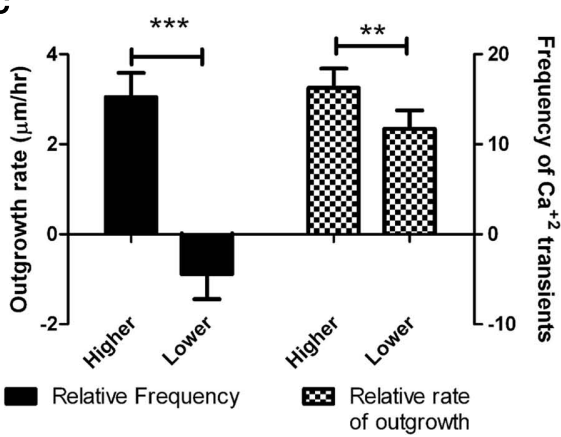

FIGURE 3 | BayK induced calcium transients, which persist throughout long term treatments, are correlated in their frequencies with process outgrowth. (A) Phase images of a cortical neuron treated with BayK in the bath for $12 \mathrm{~h}$. At the end of the imaging session the process at right (black arrowhead) had extended, whereas the process at left (red arrowhead) retracted. (B) After phase imaging, cortical neurons were loaded with Fluo-4 and calcium activity was measured. The extending process in (A) (black) showed high levels of calcium activity compared with the retracting process (red). (C) Quantifications from 16 experiments imaging calcium activity after long term BayK treatments revealed correlations between BayK induced differential calcium activity and process outgrowth. At left comparisons between processes from the same axon showed that those with higher frequency calcium transients had higher rates of outgrowth, whereas those with lower frequencies retracted $\left(n=22\right.$ comparisons; ${ }^{* *} p<0.001$, paired $t$ test). At right processes that extended faster had higher frequencies of calcium transients ( $n=26$ comparisons; ${ }^{* *} p<0.01$, paired $t$ test). Scale bar, $20 \mu \mathrm{m}$. (Reprinted from Hutchins and Kalil, 2008; with permission from the Society for Neuroscience). retraction of axonal processes, may be regulated by $\mathrm{Ca}^{2+}$ transients at optimal frequencies (Eshete and Fields, 2001). For rapid extension of cortical axons and branches the optimal frequencies of spontaneous and induced localized $\mathrm{Ca}^{2+}$ transients were similar across experiments (Hutchins and Kalil, 2008). Thus, one role for localized $\mathrm{Ca}^{2+}$ signaling may be to restrict $\mathrm{Ca}^{2+}$ transients at optimal frequencies to local regions of the axon. Local $\mathrm{Ca}^{2+}$ signaling would thereby regulate extension and retraction of axonal processes in response to environmental growth and guidance cues.

Although competition between different branches of a single axon has now been well-documented (Ruthel and Banker, 1999; Singh and Miller, 2005; Hutchins and Kalil, 2008), the competitive signaling mechanisms initiated by localized calcium activity remain mysterious. An early hypothesis of the mechanism of competitive axon growth proposed that actin ruffles flowing distally along the axon mediated competition among axonal processes (Ruthel and Banker, 1999). These actin-rich axon waves are important in promoting axon outgrowth and branching (Flynn et al., 2009). However, while disrupting actin dynamics with cytochalasin reduced rates of axon growth, the competition between processes remained intact (Ruthel and Hollenbeck, 2000). An alternative hypothesis that branches compete for mitochondria transported along the axon arose from the observation that mitochondria selectively invade axons showing robust growth (Morris and Hollenbeck, 1993). However, subsequent investigation showed that mitochondria follow, rather than precede, competitive outgrowth among axonal processes (Ruthel and Hollenbeck, 2003). More recently, it was shown that cAMP and cGMP promote or inhibit, respectively, axon formation and that these signals mutually inhibit one another (Shelly et al., 2010). This cAMP signaling in one neurite initiates a long-distance cGMP signal that hydrolyzes cAMP in all other neurites, leading to competition and the establishment of a single axon. Since calcium signaling can activate cAMP signaling (Gorbunova and Spitzer, 2002), one possibility is that the localized calcium transients that initiate competition among axonal processes (Hutchins and Kalil, 2008) do so through long-distance cyclic nucleotide signals. Supporting this possibility, recent experiments demonstrated that netrin-evoked calcium transients, of the type that promote growth cone advance, drive cAMP signals in the growth cone center (Nicol et al., 2011). Despite this promising avenue of research, it is still not known how such long-distance signals propagate or whether competitive outgrowth reflects competition for scarce resources or a purely signaling-based mechanism for restricting growth to certain branches. One possible mechanism for propagating longdistance signals is an inhibitory autocrine signal similar to that which mediates competition between neighboring axons (Singh et al., 2008). This mechanism could lead to signaling-based competition through mutual inhibition of growth between different branches from the same axon (Hutchins, 2010). However, another 
possibility is that these signaling events direct the transport of rate-limiting resources such as tubulin to the "winning" branch and thereby deprive other branches of the opportunity to grow (Butz et al., 2009; van Ooyen, 2011). The selective growth and retraction of branches is a fundamental mechanism of establishing connectivity in the mammalian CNS (McLaughlin and O'Leary, 2005). Elucidating the mechanisms of competitive axon growth will therefore be crucial for our understanding of the wiring principles of the cerebral cortex.

\section{Wnt5a PROMOTES CORTICAL AXON OUTGROWTH AND REPULSION BY DIFFERENTIAL SIGNALING}

In addition to positive guidance cues that attract cortical axons and promote their branching, repulsive guidance cues that repel growing axons are equally important in shaping the formation of cortical connections. Recently, the morphogen Wnt5a was identified as an axon guidance cue (Salinas and Zou, 2008) that repels growing cortical axons during development (Zou and Lyuksyutova, 2007). Wnts play important roles in embryogenesis such as determination of cell fate, patterning, migration, and differentiation (Ciani and Salinas, 2005). Wnt5 was first identified in Drosophila as a repulsive guidance cue for commissural axons (Yoshikawa et al., 2003). In the mouse corticospinal pathway a high to low gradient of Wnt5a in vivo acts as a repulsive cue for cortical axons (Liu et al., 2005) through the Wnt5a receptor Ryk (an atypical tyrosine kinase receptor). In vitro a source of secreted Wnt5a inhibited axon outgrowth from cortical explants facing a Wnt5a gradient. Since Wnt5a is expressed in a high to low gradient surrounding the corticospinal tract, these results (Liu et al., 2005) suggested that cortical axons must navigate down the spinal cord through a high to low inhibitory Wnt5a gradient. In the embryonic mouse corpus callosum Keeble et al. (2006) also demonstrated the repulsive effects of $\mathrm{Wnt} 5 \mathrm{a}$ on cortical axon outgrowth. In vitro axons extending from cortical explants were repelled away from a source of Wnt5a but this repulsive effect was abolished in cortex from mutant mice lacking the Ryk receptor. In vivo the corpus callosum is surrounded by a gradient of Wnt5a secreted by midline glial structures, the indusium griseum, and glial wedge (Keeble et al., 2006; Lindwall et al., 2007). In Ryk knockout mice callosal axons, which normally express Ryk receptors during development, were able to cross the midline but became misrouted after the midline and were thus unable to project away from it and formed abnormal bundles on the contralateral side. How a repulsive axon guidance cue could promote the growth of cortical axons down the spinal cord and across the corpus callosum was still a mystery.

To elucidate the mechanisms by which Wnt5a could regulate axon outgrowth of developing cortical pathways (Li et al., 2009) we bath applied Wnt5a to dissociated cortical neurons. This doubled axon outgrowth rates over 3 day in culture and in $1 \mathrm{~h}$ increased axon outgrowth fourfold. To determine whether a point source of Wnt5a could repel cortical axons, we used a turning assay in which axons were exposed to a gradient of Wnt5a. Control axons in BSA gradients showed no preference in turning toward or away from the BSA source. In contrast (Figure 4), axons consistently turned away from a source of Wnt5a by reorienting their growth cones in as little as $20 \mathrm{~min}$. However, growth cones remained motile with no obvious collapse. Surprisingly, axons turning away from the Wnt5a source also increased their growth rates by $50 \%$. This simultaneous repulsion and increased axon outgrowth in response to Wnt5a could explain how a Wnt5a gradient in vivo propels the extension of cortical axons by a repulsive mechanism.

What signaling pathways mediate responses of cortical axons to Wnt5a? Wnts exert their effects through both Ryk and Frizzled (Fz) receptors (Salinas and Zou, 2008) which are expressed on developing hamster cortical neurons (Li et al., 2009) at P0-P3 when axons are projecting into corticospinal and callosal pathways (Reh and Kalil, 1981; Norris and Kalil, 1992). Early postnatal mouse cerebral cortex also expresses seven different Fz receptors (Shimogori et al., 2004). In Fz3 knockout mice cortical axon tracts including the corpus callosum and corticospinal pathway failed to develop (Wang et al., 2002) providing the first evidence that Fz signaling plays an important role in cortical axon development. We blocked or knocked down Ryk receptors which prevented increased cortical axon outgrowth by Wnt5a (Li et al., 2009). In contrast, blocking Wnt/Fz interactions (Rodriguez et al., 2005), did not prevent increased axon outgrowth (Figure 5). These results showed that Wnt5a increases in axon outgrowth are mediated by Ryk but not Fz receptors whereas knocking down Ryk and blocking Fz receptors prevents Wnt5a repulsive turning (Figure 5). Thus both Ryk and Fz receptors mediate repulsive cortical axon guidance by Wnt5a. $\mathrm{Fz}$ receptors are involved in $\mathrm{Wnt} /$ calcium signaling during morphogenic events and in cell motility (Kohn and Moon, 2005) but the involvement of $\mathrm{Wnt} / \mathrm{calcium}$ signaling in axon growth and guidance are unknown. Further, the downstream components of Ryk signaling have not been identified. Wnt5a induced global or local $\mathrm{Ca}^{2+}$ transients involving both $\mathrm{Ryk}$ and $\mathrm{Fz}$ receptor types (Li et al., 2009). The source of intracellular calcium is important for its function (Henley and Poo, 2004; Gomez and Zheng, 2006) and $\mathrm{Ca}^{2+}$ release from intracellular stores regulates axon growth and guidance (Ooashi et al., 2005; Jacques-Fricke et al., 2006). To determine the role of this $\mathrm{Ca}^{2+}$ signaling mechanism in effects of Wnt5a we blocked $\mathrm{IP}_{3}$ receptors on the endoplasmic reticulum which blocked calcium release from intracellular stores. This partially attenuated Wnt5a induced $\mathrm{Ca}^{2+}$ activity. Calcium influx through TRP (transient receptor potential) channels also plays an important role during axon growth and guidance ( $\mathrm{Li}$ et al., 2005; Shim et al., 2005; Wang and Poo, 2005). We therefore blocked TRP channels, which also partially inhibited Wnt5a induced calcium activity. Simultaneously blocking calcium release from stores and calcium influx through TRP channels virtually silenced Wnt5a induced calcium activity. Blocking $\mathrm{Ca}^{2+}$ release from stores prevented Wnt5a induced increase in axon outgrowth but the same axons showed repulsive turning away from the source of Wnt5a (Li et al., 2009). In contrast, blocking TRP channels prevented both increased axon outgrowth and repulsion by Wnt5a. As in netrin-1 calcium signaling (Tang and Kalil, 2005) CaMKII is an essential downstream component of Wnt5a/calcium signaling. These results suggest a model (Figure 6) in which increased axon outgrowth induced by Wnt5a involves activation of Ryk receptors, calcium release from intracellular stores and activation of CaMKII signaling. Growth cone repulsion by a gradient of Wnt5a is mediated by both Ryk and $\mathrm{Fz}$ receptors and requires calcium entry through TRP channels but not calcium release from intracellular stores. In support of our results that different calcium 

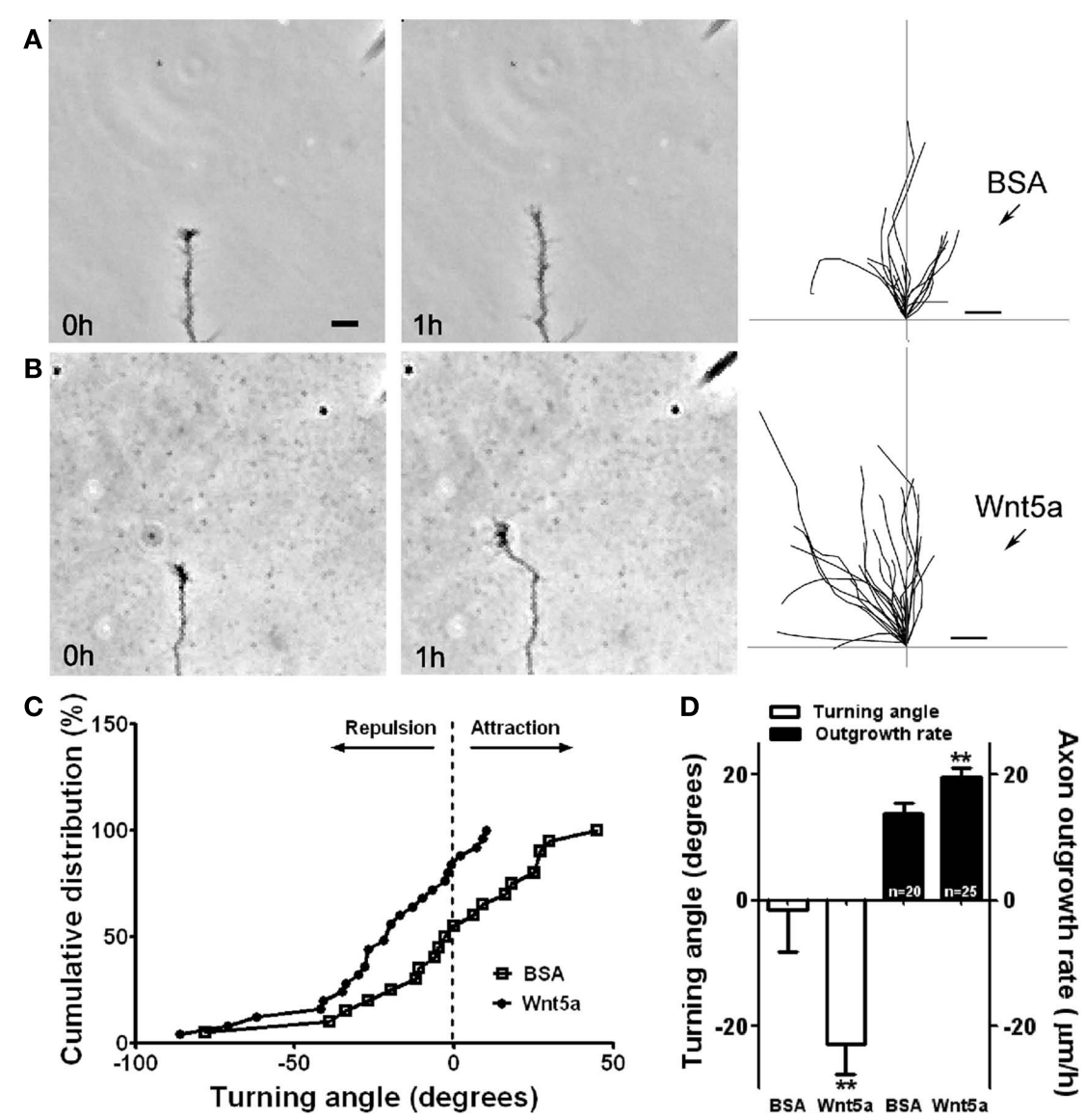

FIGURE 4 | Wnt5a gradients simultaneously increase axon outgrowth and induce repulsive axon turning. (A,B) Time lapse images of a cortical axon and its growth cone at the beginning and end of a 1-h exposure to a point source of BSA (control) (A) or $10 \mu \mathrm{g} / \mathrm{ml} \mathrm{Wnt5a}$ (B) emitted by the pipette at right. The axon extends straight in the BSA gradient but is repelled by the Wnt5a gradient. Right, tracings of trajectories of individual axons extending during $1 \mathrm{~h}$ exposure to BSA ( $n=20$ axons) or Wnt5a gradients ( $n=25$ axons) showing random turning in the presence of BSA and repulsive turning in response to Wnt5a. Note that axons repelled by Wnt5a are also significantly longer. (C) Cumulative distribution of turning angles of axons showing random turning in BSA and repulsive turning in Wnt5a gradients. (D) Bar graphs comparing average turning angles and average axon outgrowth rates in BSA and Wnt5a. In all experiments, neurons were obtained from P2 pups and cultured for 2-3 day before the turning assays. Scale bar, $5 \mu \mathrm{m}$. In all histograms, ${ }^{* *} p<0.01$. (Reprinted from Li et al., 2009; with permission from the Society for Neuroscience). signaling mechanisms (perhaps in different regions of the growth cone) mediate Wnt5a axon growth vs. guidance behaviors, a recent study (Nicol et al., 2011) showed that in Xenopus spinal neurons different calcium and cyclic AMP signaling localized to central vs. filopodial growth cone regions mediate netrin-1 evoked axon outgrowth vs. steering. Thus Wnt5a can simultaneously activate different receptors and different calcium signaling pathways at the growth cone to elicit growth and guidance behaviors required for wiring of cortical circuits during development.

\section{AXON GROWTH AND GUIDANCE IN THE CORPUS CALLOSUM IS REGULATED BY Wnt/CALCIUM SIGNALING}

Thus far we have discussed mechanisms of axon growth and guidance in dissociated cortical neurons. However, it is important to test the relevance of these mechanisms in a more complex cellular environment that mimics development in vivo. For example, in dissociated cultures knocking down CaMKI decreased hippocampal and cortical axon elongation (Ageta-Ishihara et al.,
2009; Davare et al., 2009; Neal et al., 2010) whereas knockdown of CaMKI in vivo during activity-dependent cortical wiring decreased callosal axon branching into cortex without affecting rates of callosal axon elongation (Ageta-Ishihara et al., 2009). To test the in vivo function of $\mathrm{Wnt} /$ calcium signaling mechanisms we used living slices of developing hamster sensorimotor cortex that contained the entire callosal pathway (Hutchins et al., 2011). This permitted live cell imaging of intact callosal axons extending along their entire trajectory (Halloran and Kalil, 1994). We electroporated plasmids, encoding the cytoplasmic fluorescent marker DsRed2, into one hemisphere of cortical slices from P0 brains and after $48 \mathrm{~h}$ used confocal microscopy to image fluorescently labeled callosal axons (Figure 7), which exhibited continual growth cone motility and forward advance during the 90-min imaging sessions. Importantly, axons before the midline (precrossing) advanced more slowly than those that have crossed the midline (postcrossing). To determine whether callosal axons expressed $\mathrm{Ca}^{2+}$ transients correlated with rates of 
axon outgrowth we electroporated a genetically encoded calcium indicator (GCaMP2) into one hemisphere and measured frequencies of $\mathrm{Ca}^{2+}$ transients in pre- and post-crossing axons for up to $30 \mathrm{~min}$. (Figure 8). Calcium transients were expressed in axons and growth cones (Figure 8) in frequencies closely related to rates of callosal axon outgrowth. Thus higher frequencies of $\mathrm{Ca}^{2+}$

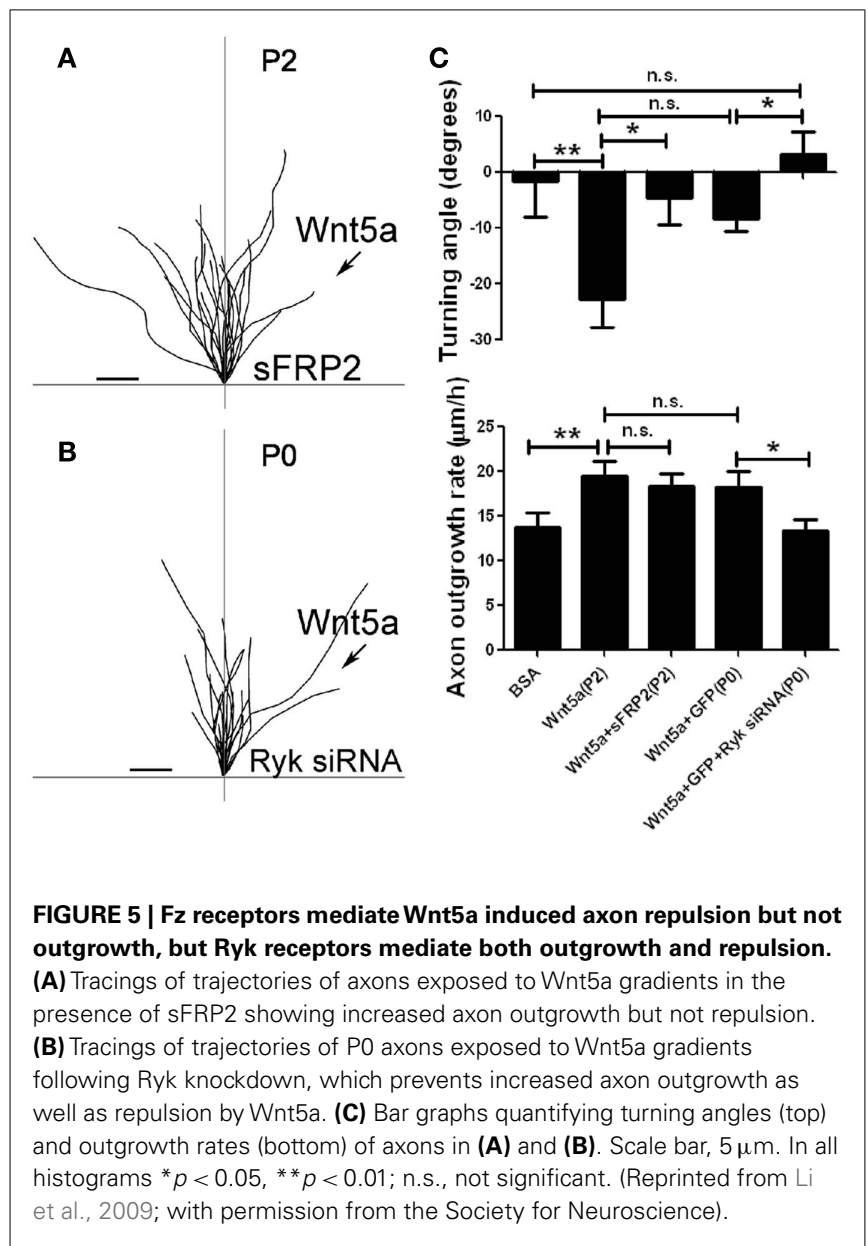

transients are well correlated with higher rates of callosal axon outgrowth, similar to our results for dissociated cortical neurons (Hutchins and Kalil, 2008).

The corpus callosum is surrounded by a Wnt5a gradient secreted by midline glial structures (Keeble et al., 2006). Since callosal axons express $\mathrm{Ca}^{2+}$ transients correlating with rates of axon outgrowth we reasoned that in the corpus callosum the Wnt5a gradient could mediate axon growth and guidance by calcium signaling mechanisms. To determine whether $\mathrm{Ca}^{2+}$ release from

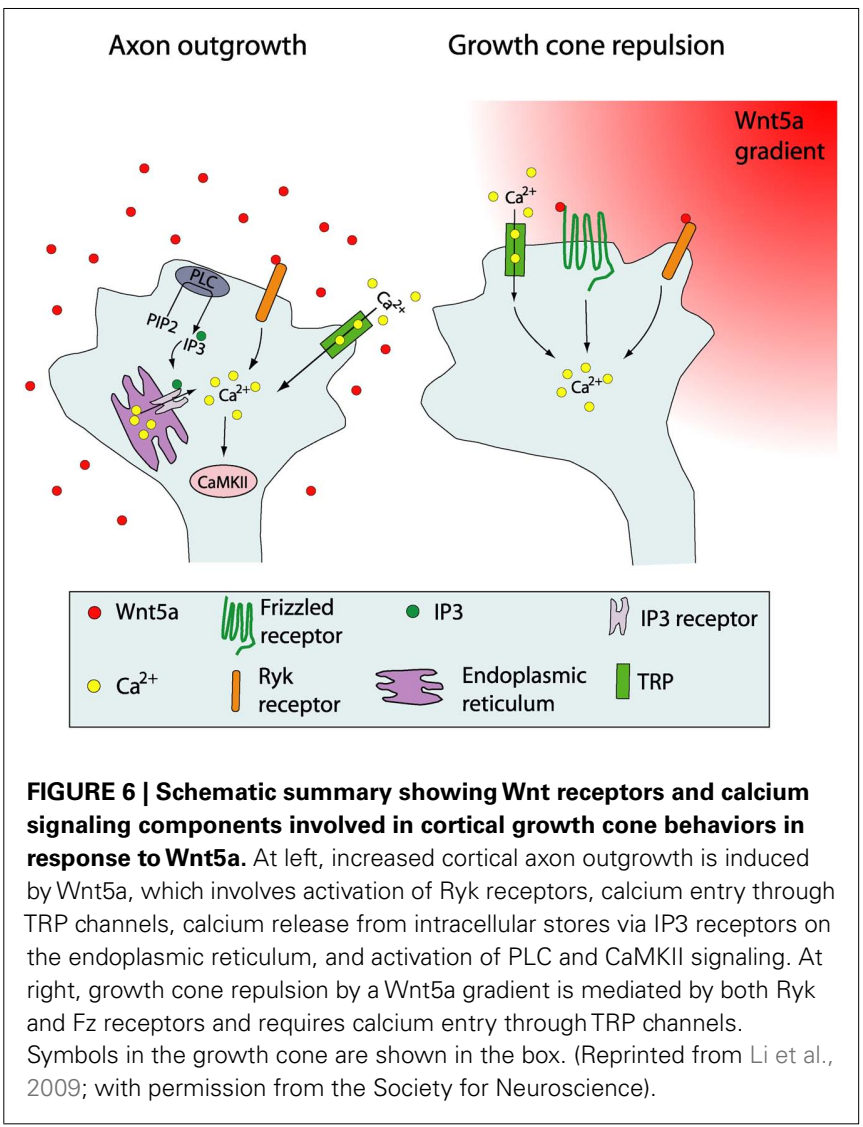

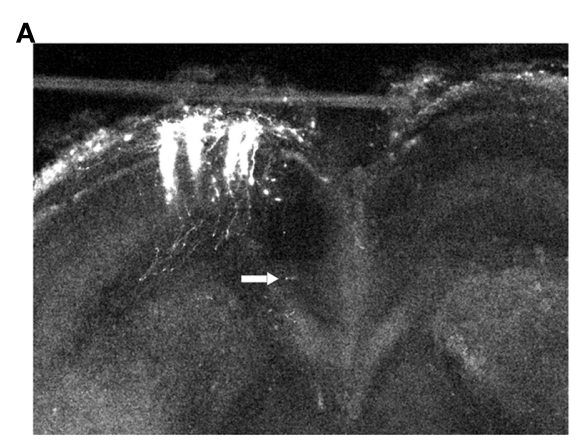

B

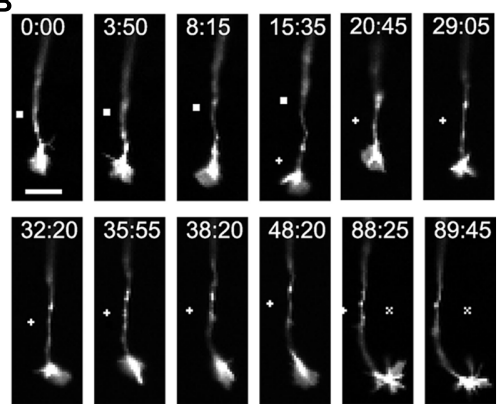

FIGURE 7 | Imaging of individual callosal axons and their growth cones extending through the corpus callosum. (A) A low power confocal image of a cortical slice at 3DIV, after electroporation of cortical neurons with DsRed2 in the slice from P0 sensorimotor hamster cortex. Individual efferent axons are clearly visible. Arrow indicates location of the cortical growth cone imaged at higher power in the time lapse sequence in (B) which shows filopodia and lamellipodia of growth cones during outgrowth and turning. Times are in minutes and seconds. Scale bar, $10 \mu \mathrm{m}$. Symbols show reference points. (Reprinted from Hutchins et al., 2011). 


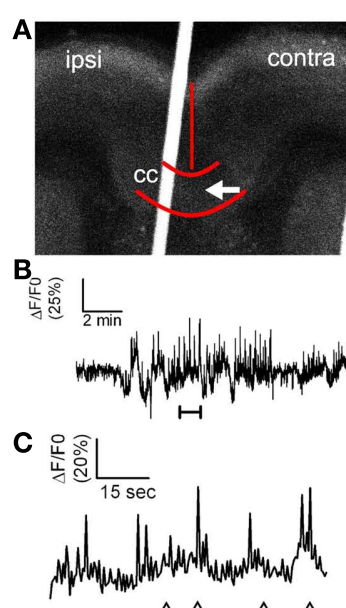

$\Delta \triangle \triangle \Delta$

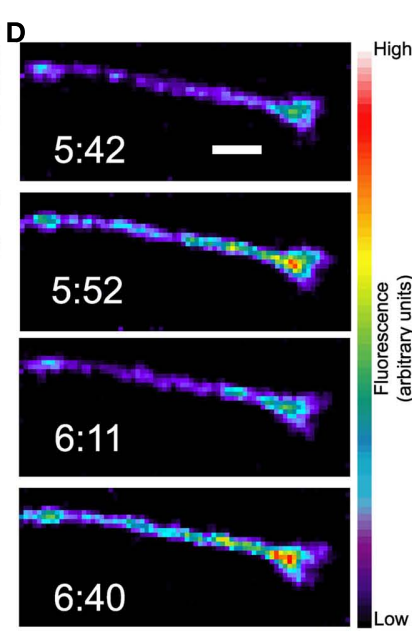

E.
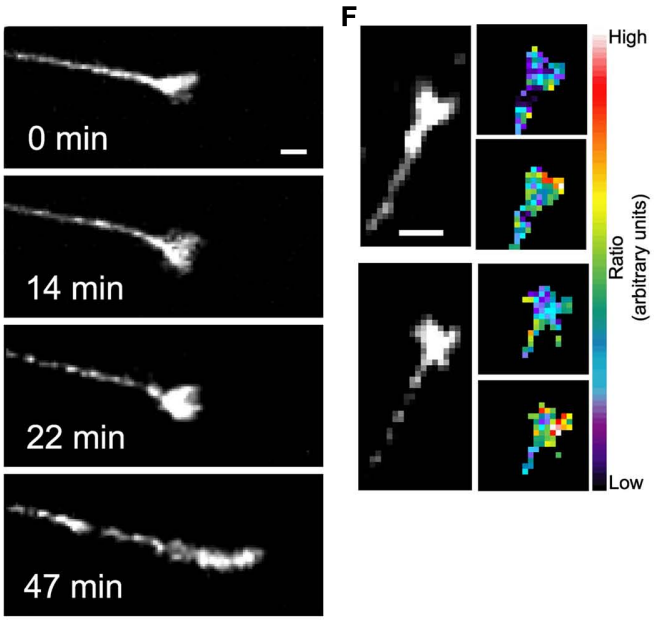

G

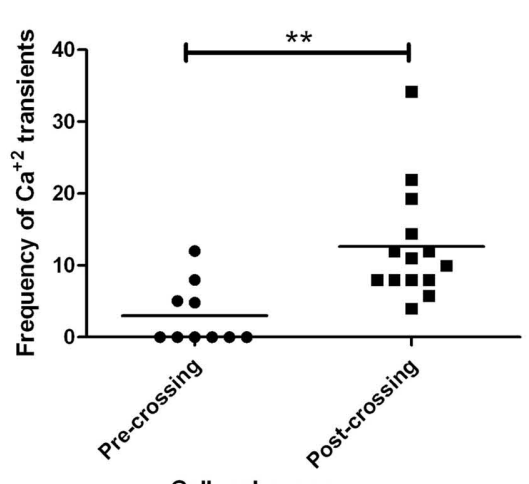

I Callosal axons
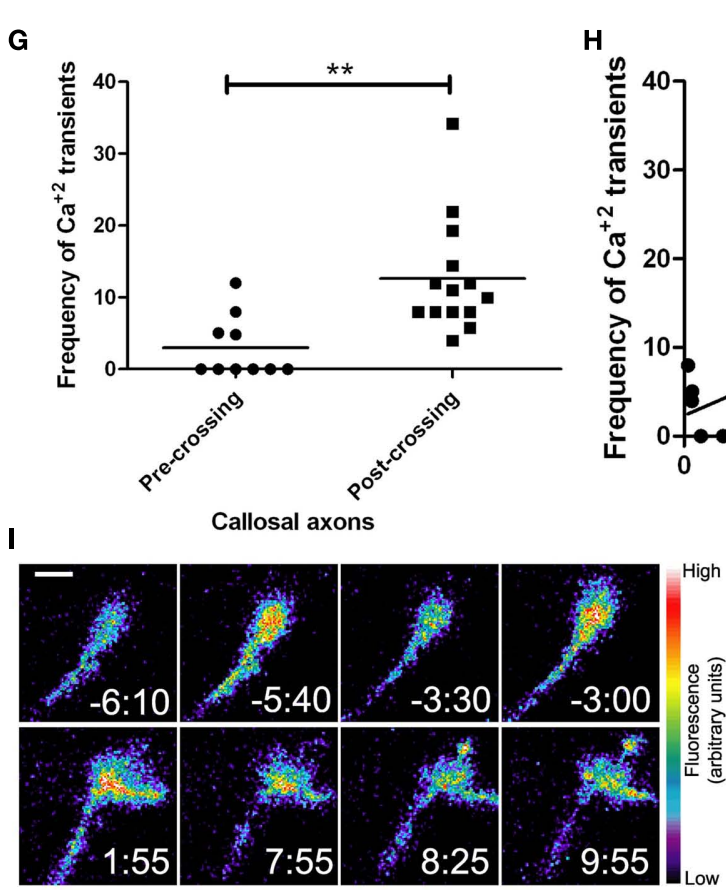

FIGURE 8 | Callosal axons express spontaneous calcium transients positively correlated with rates of axon outgrowth. (A) A coronal cortical slice electroporated with GCaMP2 plasmids into the left cortex. Arrow indicates postcrossing (contra) position of the growth cone imaged in (D). Borders of the callosum (cc) and the midline are outlined in red. (B) Tracing of calcium activity measured by changes in GCaMP2 fluorescence over baseline. Calcium activity increases after a few min. (C) Tracing of calcium activity from (B) zoomed in to the time shown in the bracket. (D) Fluorescence images of the growth cone measured in $\mathbf{( B , C )}$ at time points indicated by the arrowheads in (C). (E) Within 20 min of the onset of calcium activity shown in (B) the axon begins to advance rapidly through the contralateral callosum. (F) Examples of single calcium transients measured by ratiometric imaging of

stores plays a role in regulating callosal axon outgrowth we blocked $\mathrm{IP}_{3}$ receptors (on the endoplasmic reticulum) pharmacologically to inhibit calcium release from stores. Using live cell imaging, we measured growth of postcrossing axons since a recent study growth cones co-expressing DsRed2 and GCaMP2. (G) Plot of frequencies of calcium transients in pre- or post-crossing callosal axons, ${ }^{* *} p<0.01, t$ test. All frequencies are in transients/h. (H) Scatter plot of the frequency of calcium transients vs. the rate of axon outgrowth in individual callosal axons. The line represents the least-squares linear regression (slope significantly non-zero, $p<0.01$ ). (I) An example of spontaneous calcium transients (top row) which are attenuated by application of SKF at time 0.00 (bottom row). (J) Tracing of calcium activity in the growth cone shown in (I) before and after application of SKF. Scale bars $10 \mu \mathrm{m}$ except (I) which is $5 \mu \mathrm{m}$. Pseudocolor calibration bars indicate fluorescence intensity (D) or ratio of GCaMP2 to DsRed2

fluorescence intensities (F) in arbitrary units. (Reprinted from Hutchins et al., 2011).
(Wang et al., 2007) showed that in vivo suppression of spontaneous electrical activity in mouse callosal axons decreased rates of axon outgrowth on the postcrossing but not the precrossing side of the callosum. Treatment with blockers to $\mathrm{IP}_{3}$ receptors slowed 
axon outgrowth of postcrossing axons but their normal rates were restored after washout of the blocking reagent. However, blocking $\mathrm{Ca}^{2+}$ release from stores had no effect on guidance of callosal axons whose trajectories (measured from images obtained at the beginning and end of the 3-h experiments) after crossing the midline were similar to those of untreated controls. In contrast, pharmacologically blocking $\mathrm{Ca}^{2+}$ entry through TRP channels not only slowed axon outgrowth but also severely misrouted postcrossing callosal axons, which turned prematurely toward the cortical plate or turned ventrally toward the septum. We verified the involvement of calcium activity by blocking TRP channels and then measuring effects on $\mathrm{Ca}^{2+}$ transients in individual postcrossing callosal axons. As shown in Figure 8I, blocking TRP channels attenuated frequencies of $\mathrm{Ca}^{2+}$ transients to about $3 \mathrm{~h}$ compared with $12 \mathrm{~h}$ for controls, showing directly that the growth and guidance defects resulted from decreased $\mathrm{Ca}^{2+}$ activity. Furthermore, as we found in dissociated cultures, calcium signaling by store release or by $\mathrm{Ca}^{2+}$ entry through TRP channels respectively mediates axon outgrowth or both axon outgrowth and guidance in the corpus callosum.

Although these results demonstrate the requirement for calcium signaling in outgrowth and guidance of callosal axons, it was important to show the specific involvement of Wnt5a signaling. In vivo knockout mice lacking the Wnt5a Ryk receptor showed guidance errors in callosal axons only after they crossed the midline (Keeble et al., 2006). This is consistent with the finding that mouse callosal axons do not respond to Wnt5a until the age when they cross the midline (E 16) and begin to express the Ryk receptor (Keeble et al., 2006). However, these experiments did not address signaling mechanisms downstream of Ryk receptors. Therefore, we knocked down the Ryk receptor by electroporation of Ryk siRNA in a small number of axons and then analyzed effects on DsRed2 labeled callosal axons in living cortical slices (Hutchins et al., 2011). Ryk knockdown slowed the growth rate of postcrossing callosal axons to half their normal growth rate, but had no effect on precrossing axon growth rates, consistent with Ryk expression on callosal axons only after they cross the midline (Keeble et al., 2006). Importantly, Ryk knockdown also caused severe guidance errors in about a third of labeled postcrossing axons that deviated inappropriately toward the septum or prematurely turned toward the overlying cortex. Since we found previously ( $\mathrm{Li}$ et al., 2009) that knocking down the Ryk receptor reduced the proportion of neurons expressing $\mathrm{Ca}^{2+}$ transients in response to Wnt5a, we asked whether growth and guidance defects in callosal axons in which Ryk was knocked down resulted from interference with Wnt5a evoked calcium signaling. To address this question we coelectroporated the calcium biosensor GCaMP2 with Ryk siRNA to measure calcium activity in neurons in which RyK/Wnt signaling was disrupted. In such neurons frequencies of $\mathrm{Ca}^{2+}$ transients were reduced fourfold in postcrossing axons. Remarkably, in growth cones projecting aberrantly toward the septum, $\mathrm{Ca}^{2+}$ transients were undetectable. Taken together, these results suggest that callosal axon growth and guidance errors caused by Ryk knockdown result from attenuation of calcium activity. Since CaMKII is also a component of the Wnt/calcium signaling pathway (Li et al., 2009), we investigated its role in callosal axon growth and guidance by cortical transfection of plasmids encoding an inhibitory
CaMKII protein (Tang and Kalil, 2005). For postcrossing but not precrossing axons, this treatment slowed axon outgrowth by about half and caused dramatic axon guidance errors in which some axons projected aberrantly while others looped backward in the corpus callosum. Do these defects represent a failure of axon repulsion mechanisms mediated by Wnt5a? Using turning assays in which dissociated neurons were exposed to Wnt5a gradients, we found that neurons transfected with the CaMKII inhibitor were unable to increase their growth rates or show repulsive turning. These results suggest that CaMKII, downstream of Wnt/calcium signaling, is necessary for the outgrowth and repulsive guidance mechanisms by which Wnt5a regulates development of callosal axons.

Taken together these results, summarized in a cortical slice model of the developing corpus callosum (Figure 9), show that Wnt/calcium signaling pathways are essential for regulating callosal axon outgrowth and guidance. Nevertheless, additional growth and guidance factors are known to regulate development of the corpus callosum (Lindwall et al., 2007). For example, in addition to Wnt5a, sources of netrins, semaphorins, and Slit 2 surround the corpus callosum and their role in callosal axon guidance across the midline has been well characterized (Shu and Richards, 2001; Shu et al., 2003; Niquille et al., 2009; Piper et al., 2009). Although direct evidence for netrins in guiding callosal axons to the midline is lacking, callosal axons express the netrin-1 receptor DCC and netrin-1 knockout mice lack all forebrain commissural projections (Serafini et al., 1996). Slits are chemorepellent proteins expressed in glial structures adjacent to the callosal midline in a pattern similar to that of Wnt5a. Knockout mice lacking the Slit receptor Robo 1 display aberrant projections of callosal axons that are misdirected at the midline, suggesting that Slits may channel callosal axons into the correct pathway (Lindwall et al., 2007). Mutant mice whose axons are unable to bind semaphorins also show callosal malformations. However, our finding that inhibiting calcium signaling only affected growth of axons after but not before the midline suggests the involvement of Wnt5a signaling because cortical axons do not respond to Wnt5a until the age at which they cross the midline (Keeble et al., 2006). Although Slit 2 affects both pre- and post-crossing axons (Shu and Richards, 2001; Shu et al., 2003) our results show that interfering with Wnt/calcium/CaMKII signaling only affects postcrossing axon outgrowth.

\section{MICROTUBULE DYNAMICS REGULATE Wnt5a EVOKED AXON OUTGROWTH AND GUIDANCE}

Growth cone behaviors are regulated by reorganization and dynamics of the actin and microtubule (MT) cytoskeleton (Dent and Gertler, 2003; Lowery and Van Vactor, 2009), which can be modulated by extracellular guidance cues (Luo, 2002; Huber et al., 2003; Kalil and Dent, 2005). Stable microtubules occupy the central region of the growth cone while dynamic MTs that grow and shrink in dynamic instability actively explore the periphery (Dent et al., 1999; Dent and Kalil, 2001; Schaefer et al., 2002, 2008; Lowery and Van Vactor, 2009). Inhibiting MT dynamics can abolish growth cone turning and localized MT stabilization and de-stabilization can induce attractive turning and repulsion (Buck and Zheng, 2002), suggesting an instructive role for MTs in axon guidance. Wnt signaling increases MT stability and Wnt3a 


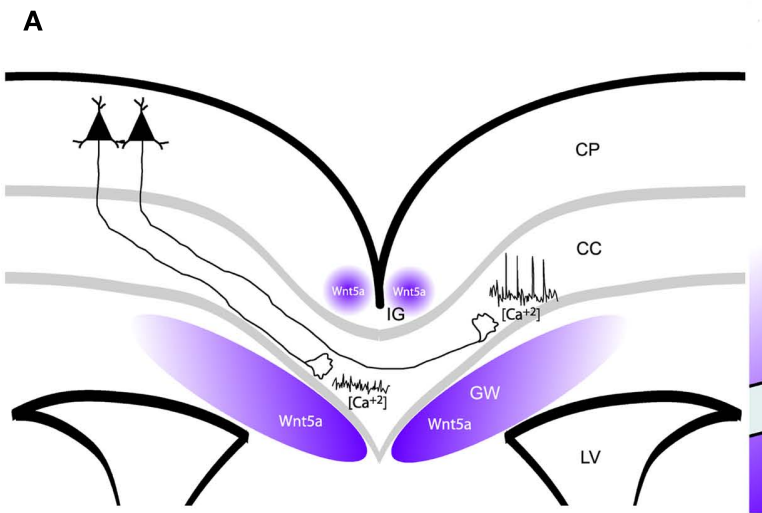

FIGURE 9 |Wnt/calcium signaling guides axon growth through the corpus callosum. (A) Schematic of a cortical slice showing cortical axons extending through the corpus callosum (cc) surrounded by Wnt5a secreted by the indusium griseum (IG) and glial wedge (GW). Axons in the contralateral callosum have high frequencies of calcium transients evoked by Wnt5a signaling, which accelerates rates of axon outgrowth. In contrast, ipsilateral axons not yet responsive to Wnt5a, have low levels of calcium activity and thus grow more slowly. LV, lateral ventricle; $\mathrm{CP}$,

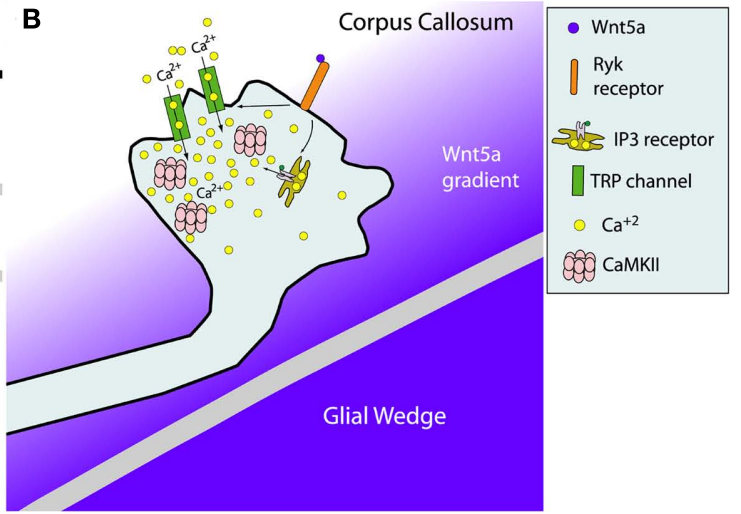

cortical plate. (B) Schematic of intracellular signaling in the postcrossing axon from (A) showing that Wnt5a gradient from the glial wedge activates RyK receptors to open IP3 receptors and TRP channels causing release of calcium from intracellular stores and calcium influx through the plasma membrane. These calcium transients activate CaMKII to accelerate axon extension and repel growth cones away from the glial wedge toward the contralateral cortex. (Reprinted from Hutchins et al., 2011). and Wnt7a specifically decrease axon length and increase growth cone size of DRG and cerebellar mossy fiber terminals (Lucas and Salinas, 1997; Hall et al., 2000) by reorganization of MTs into stable loops (Ciani and Salinas, 2005; Salinas, 2007; Purro et al., 2008). This occurs through changes in MT directionality across the growth cone rather than toward the leading edge (Purro et al., 2008). In contrast we showed that Wnt5a increases cortical axon outgrowth and induces growth cone repulsion (Li et al., 2009) but the cytoskeletal mechanisms are unknown. Our preliminary results ( $\mathrm{Li}$ et al., unpublished observations) suggest that reorganization of dynamic MTs plays a major role in increased axon outgrowth and repulsive growth cone turning induced by Wnt5a.

We first confirmed that dynamic MTs are required for the growth promoting and guidance effects of Wnt5a by applying nocodazole, an inhibitor of MT dynamics (Dent and Kalil, 2001), which prevented Wnt5a induced increases in axon outgrowth and repulsive turning in $\mathrm{Wnt} 5$ a gradients. To determine the relationship between directionality of MTs and the direction of growth cone advance induced by Wnt5a, we used high resolution fluorescence (total internal reflection fluorescence, TIRF) microscopy to image growth cones of early postnatal cortical neurons transfected with an EGFP-EB3 construct to label dynamic MT plus ends and DsRed 2 to label the cytoplasm. EB3 is a +TIP protein that binds to the growing plus ends of MTs (Nakagawa et al., 2000) and EGFPEB3 has been used to image MT dynamics in neurons (Stepanova et al., 2003). Dynamic growing MT tips were visualized as EB3 "comets" in cortical growth cones before and after bath application of Wnt5a. Wnt5a oriented dynamic MTs in the direction of axon elongation, suggesting that increased axon outgrowth by Wnt5a occurs by reorientation of dynamic MTs. We then applied Wnt5a as a gradient to investigate the possible redistribution of dynamic MTs in growth cones during repulsive turning behaviors. Preliminary results (Figure 10) showed that before application of Wnt5a
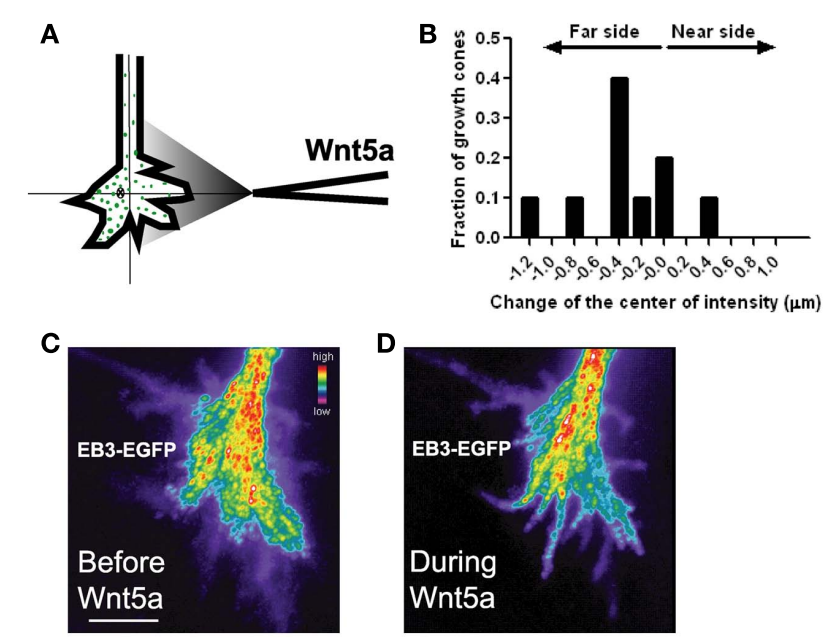

FIGURE 10 |Wnt5a gradients induce redistribution and reorganization of dynamic microtubules (MTs) in growth cones. (A) Schematic of measurement of the distribution of EB3 comets on MT plus ends in the presence of a pipette gradient of Wnt5a locally applied at the right side of the growth cone. Individual EB3 comets are detected by fluorescence (green dots) and their center of intensity (cross) is determined. (B) Localization of the center of intensity of EB3 comets evoked by a Wnt5a gradient in 10 growth cones in independent experiments including the growth cone shown in (C,D). Negative numbers indicate EB3 localization to the side of the growth cone farthest from the Wnt5a source. Change of the center of intensity $=-0.35 \pm 0.14 \mu \mathrm{m}(p<0.05$, Wilcoxon signed rank test). (C,D) Composite images of the same growth cone showing redistribution of EB3 comets over time $30 \mathrm{~min}$ before and during $30 \mathrm{~min}$ of application of the Wnt5a gradient ( $n=601$ images before Wnt5a and 601 images during Wnt5a application). The maximum intensity was computed for each pixel from the entire image stack by using algorithms in Metamorph. Using these values stacks of all images were superimposed and pixels assigned values in pseudocolor to indicate fluorescence intensity. 
EB3 comets were randomly distributed throughout the growth cone but during 30 min exposure of growth cones to Wnt5a gradients EB3 comets redistributed to the far side of the growth cone away from the Wnt5a source. These results suggest that in Wnt5a gradients asymmetric redistribution of dynamic MTs may be a first step in repulsive turning.

Gradients of guidance cues can evoke asymmetries in calcium activity (Gomez and Zheng, 2006) that are typically highest on the side of the growth cone facing a source of either an attractive or repulsive guidance cue gradient. Thus it was surprising to find that Wnt5a can evoke $\mathrm{Ca}^{2+}$ transients localized to the far side of the growth cone facing away from the Wnt5a source. The coincidence of MT and $\mathrm{Ca}^{2+}$ asymmetries led us to investigate a possible relationship between asymmetries in $\mathrm{Ca}^{2+}$ transients and the redistribution of dynamic MTs in growth cones exposed to Wnt5a gradients. One candidate link is the MT associated protein tau which binds to MTs to increase their stability (Dehmelt and Halpain, 2005). CaMKII phosphorylates tau at the MT binding site (Litersky et al., 1996; Sironi et al., 1998). This leads to detachment of tau from MTs and thus decreased MT stability and increased MT dynamics (Biernat et al., 1993). Furthermore, Wnt5a is known to activate CaMKII (Kuhl et al., 2000). Together these findings led us to investigate tau as a possible link between calcium signaling and dynamic MTs. To determine whether tau is required for the growth promoting effects of Wnt5a on cortical axons, we knocked down tau with siRNAs which prevented Wnt5a from increasing rates of cortical axon outgrowth. Importantly, this treatment also prevented growth cone repulsion in Wnt5a gradients. We then co-transfected cortical neurons with EGFP-EB3 and tau siRNA, and imaged dynamic MTs by following EB3 comets in growth cones. Remarkably, we found that tau knockdown prevented the redistribution of dynamic MTs toward the far side of the growth cone facing away from the Wnt5a gradient. As shown in Figure 11 dynamic MTs remain randomly distributed throughout the growth cones as in untreated controls. Since calcium activity is required for the growth and guidance effects of Wnt5a on cortical axons in vitro (Li et al., 2009) and within the corpus callosum (Hutchins et al., 2011) these results suggest a relationship, possibly mediated by tau, between Wnt5a evoked calcium signaling and the redistribution of dynamic MTs during Wnt5a evoked cortical growth cone repulsion.

\section{FUTURE DIRECTIONS}

This review has emphasized the central role of intracellular calcium signaling in transducing the effects of guidance molecules on growth and guidance of cortical axons during development. However, many questions remain unanswered. First, the mechanisms by which guidance cues evoke calcium transients localized to small regions of axons and growth cones are not well understood. Second, how competitive mechanisms, mediated by calcium signaling, favor the growth of one cortical axon process over another requires further study. Third, the relationship between calcium activity and cytoskeletal dynamics leading to cortical axon branching, outgrowth and guidance remains obscure. Fourth, effects of guidance cues on cytoskeletal reorganization leading to specific growth cone behaviors are only beginning to be addressed. Finally, it is not always clear that axon growth and guidance mechanisms identified in tissue culture are applicable to development in vivo. Thus a major challenge will be to elucidate how axons of neurons in the complex in vivo environment of the cerebral cortex can integrate multiple extracellular guidance cues and intracellular signaling pathways necessary for appropriate axon outgrowth, guidance, and branching during wiring of cortical circuits.

\section{ACKNOWLEDGMENTS}

The work from our laboratory was funded by National Institutes of Health Grant NSO14428 and grants from the Whitehall
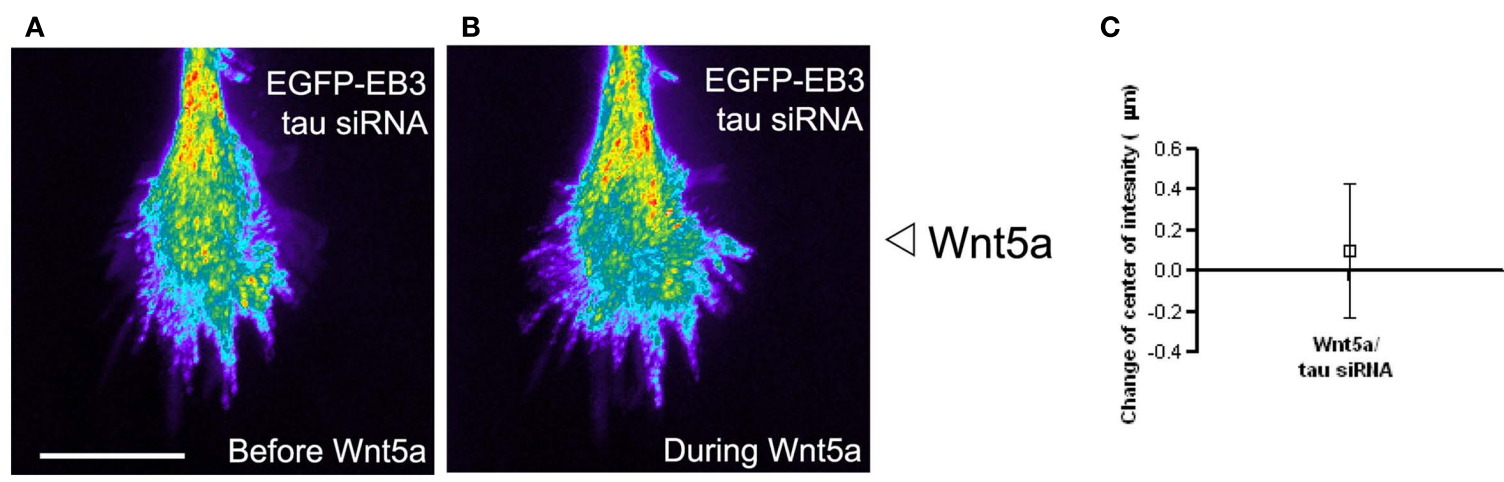

FIGURE 11 |The microtubule associated protein tau is required for Wnt5a induced redistribution of dynamic MTs in growth cones. (A,B) Composite images showing the redistribution of EB3 comets in the same growth cone over time $30 \mathrm{~min}$ before and during $30 \mathrm{~min}$ of application of the Wnt5a gradient ( $n=361$ images before Wnt5a and 601 images during Wnt5a application). The maximum intensity was computed for each pixel from the entire image stack by using algorithms in Metamorph. Using these values stacks of all images were superimposed and pixels assigned values in pseudocolor to indicate fluorescence intensity. Wnt5a was locally applied as a gradient at the right side of the growth cone. In this growth cone tau was knocked down with siRNA, which prevented the asymmetric redistribution of EB3 comets during exposure of the growth cone to Wnt5a gradients. (C) Localization of the center of intensity of EB3 comets evoked by Wnt5a gradients in four growth cones in independent experiments including the growth cone shown in $(\mathbf{A}, \mathbf{B})$. Quantitative analysis shows that Wnt5a fails to induce redistribution of EB3 comets to the far side of the growth cone after tau knockdown with siRNA (change in center of intensity not different from 0 . $p>0.05$, Wilcoxon signed rank test). 
Foundation and the University of Wisconsin Graduate School (to Katherine Kalil), a Herman Shapiro fellowship (to Li Li), and National Research Service Award GM801642 (to Bruce Ian
Hutchins). Bruce Ian Hutchins is currently supported by the Intramural Research Program of NINDS and the Pharmacology Research Associate Program of NIGMS.

\section{REFERENCES}

Ageta-Ishihara, N., Takemoto-Kimura, S., Nonaka, M., Adachi-Morishima, A., Suzuki, K., Kamijo, S., Fujii, H., Mano, T., Blaeser, F., Chatila, T. A., Mizuno, H., Hirano, T., Tagawa, Y., Okuno, H., and Bito, H. (2009). Control of cortical axon elongation by a GABA-driven $\mathrm{Ca} 2+/$ calmodulin-dependent protein kinase cascade. J. Neurosci. 29, 13720-13729.

Bagnard, D., Lohrum, M., Uziel, D., Puschel, A. W., and Bolz, J. (1998). Semaphorins act as attractive and repulsive guidance signals during the development of cortical projections. Development 125 , 5043-5053.

Bastmeyer, M., and O'Leary, D. D. (1996). Dynamics of target recognition by interstitial axon branching along developing cortical axons. $J$. Neurosci. 16, 1450-1459.

Biernat, J., Gustke, N., Drewes, G., Mandelkow, E. M., and Mandelkow, E. (1993). Phosphorylation of Ser262 strongly reduces binding of tau to microtubules: distinction between PHF-like immunoreactivity and microtubule binding. Neuron 11, 153-163.

Borodinsky, L. N., O'Leary, D., Neale, J. H., Vicini, S., Coso, O. A., and Fiszman, M. L. (2003). GABAinduced neurite outgrowth of cerebellar granule cells is mediated by $\mathrm{GABA}(\mathrm{A})$ receptor activation, calcium influx and CaMKII and erk1/2 pathways. J. Neurochem. 84, 1411-1420.

Buck, K. B., and Zheng, J. Q. (2002). Growth cone turning induced by direct local modification of microtubule dynamics. J. Neurosci. 22, 9358-9367.

Butz, M., Worgotter, F., and van Ooyen, A. (2009). Activity-dependent structural plasticity. Brain Res. Rev. 60, 287-305.

Cancedda, L., Fiumelli, H., Chen, K., and Poo, M. M. (2007). Excitatory GABA action is essential for morphological maturation of cortical neurons in vivo. J. Neurosci. 27, 5224-5235.

Ciani, L., and Salinas, P. C. (2005). WNTs in the vertebrate nervous system: from patterning to neuronal connectivity. Nat. Rev. Neurosci. 6, 351-362.

Cullen, P. J., and Lockyer, P. J. (2002). Integration of calcium and Ras signaling. Nat. Rev. Mol. Cell Biol. 3, 339-348.

Davare, M. A., Fortin, D. A., Saneyoshi, T., Nygaard, S., Kaech, S., Banker, G., Soderling, T. R., and Wayman, G. A. (2009). Transient receptor potential canonical 5 channels activate $\mathrm{Ca} 2+/$ calmodulin kinase Igamma to promote axon formation in hippocampal neurons. J. Neurosci. 29, 9794-9808.

Dehmelt, L., and Halpain, S. (2005). The MAP2/Tau family of microtubuleassociated proteins. Genome Biol. 6, 204.

Dent, E. W., Barnes, A. M., Tang, F., and Kalil, K. (2004). Netrin-1 and semaphorin $3 \mathrm{~A}$ promote or inhibit cortical axon branching, respectively, by reorganization of the cytoskeleton. $J$. Neurosci. 24, 3002-3012.

Dent, E. W., Callaway, J. L., Szebenyi, G., Baas, P. W., and Kalil, K. (1999). Reorganization and movement of microtubules in axonal growth cones and developing interstitial branches. J. Neurosci. 19, 8894-8908.

Dent, E. W., and Gertler, F. B. (2003). Cytoskeletal dynamics and transport in growth cone motility and axon guidance. Neuron 40, 209-227.

Dent, E. W., and Kalil, K. (2001). Axon branching requires interactions between dynamic microtubules and actin filaments. J. Neurosci. 21, 9757-9769.

Dent, E. W., Tang, F., and Kalil, K. (2003). Axon guidance by growth cones and branches: common cytoskeletal and signaling mechanisms. Neuroscientist 9, 343-353.

Dickson, B. J. (2002). Molecular mechanisms of axon guidance. Science 298, 1959-1964.

Eshete, F., and Fields, R. D. (2001). Spike frequency decoding and autonomous activation of $\mathrm{Ca} 2+-$ calmodulin-dependent protein kinase II in dorsal root ganglion neurons. J. Neurosci. 21, 6694-6705.

Fink, C. C., Bayer, K. U., Myers, J. W., Ferrell, J. E. Jr., Schulman, H., and Meyer, T. (2003). Selective regulation of neurite extension and synapse formation by the beta but not the alpha isoform of CaMKII. Neuron 39, 283-297.

Flynn, K. C., Pak, C. W., Shaw, A. E., Bradke, F., and Bamburg, J. R. (2009). Growth cone-like waves transport actin and promote axonogenesis and neurite branching. Dev. Neurobiol. 69, 761-779.

Gomez, T. M., and Zheng, J. Q. (2006). The molecular basis for calciumdependent axon pathfinding. Nat. Rev. Neurosci. 7, 115-125.

Gorbunova, Y. V., and Spitzer, N. C. (2002). Dynamic interactions of cyclic AMP transients and spontaneous $\mathrm{Ca}(2+)$ spikes. Nature 418 , 93-96.

Hall, A. C., Lucas, F. R., and Salinas, P. C. (2000). Axonal remodeling and synaptic differentiation in the cerebellum is regulated by WNT-7a signaling. Cell 100, 525-535.

Halloran, M. C., and Kalil, K. (1994). Dynamic behaviors of growth cones extending in the corpus callosum of living cortical brain slices observed with video microscopy. J. Neurosci. 14, 2161-2177.

Harter, P. N., Bunz, B., Dietz, K., Hoffmann, K., Meyermann, R., and Mittelbronn, M. (2010). Spatiotemporal deleted in colorectal cancer (DCC) and netrin-1 expression in human foetal brain development. Neuropathol. Appl. Neurobiol. 36, 623-635.

Henley, J., and Poo, M. M. (2004). Guiding neuronal growth cones using $\mathrm{Ca} 2+$ signals. Trends Cell Biol. 14, 320-330.

Hong, K., Nishiyama, M., Henley, J., Tessier-Lavigne, M., and Poo, M. (2000). Calcium signaling in the guidance of nerve growth by netrin1. Nature 403, 93-98.

Hua, J. Y., Smear, M. C., Baier, H., and Smith, S. J. (2005). Regulation of axon growth in vivo by activity-based competition. Nature 434, 1022-1026.

Huber, A. B., Kolodkin, A. L., Ginty, D. D., and Cloutier, J. F. (2003). Signaling at the growth cone: ligandreceptor complexes and the control of axon growth and guidance. Annu. Rev. Neurosci. 26, 509-563.

Hudmon, A., and Schulman, H. (2002). Neuronal CA2+/calmodulindependent protein kinase II: the role of structure and autoregulation in cellular function. Annu. Rev. Biochem. 71, 473-510.

Hutchins, B. I. (2010). Competitive outgrowth of neural processes arising from long-distance cAMP signaling. Sci. Signal. 3, jcl.

Hutchins, B. I., and Kalil, K. (2008). Differential outgrowth of axons and their branches is regulated by localized calcium transients. J. Neurosci. 28, 143-153.

Hutchins, B. I., Li, L., and Kalil, K. (2011). Wnt/calcium signaling mediates axon growth and guidance in the developing corpus callosum. Dev. Neurobiol. 71, 269-283.

Jacques-Fricke, B. T., Seow, Y., Gottlieb, P. A., Sachs, F., and Gomez, T. M. (2006). Ca2+ influx through mechanosensitive channels inhibits neurite outgrowth in opposition to other influx pathways and release from intracellular stores. J. Neurosci. 26, 5656-5664.

Kalil, K., and Dent, E. W. (2005). Touch and go: guidance cues signal to the growth cone cytoskeleton. Curr. Opin. Neurobiol. 15, 521-526.

Kalil, K., Szebenyi, G., and Dent, E. W. (2000). Common mechanisms underlying growth cone guidance and axon branching. J. Neurobiol. 44, 145-158.

Keeble, T. R., Halford, M. M., Seaman, C., Kee, N., Macheda, M., Anderson, R. B., Stacker, S. A., and Cooper, H. M. (2006). The Wnt receptor Ryk is required for Wnt5a-mediated axon guidance on the contralateral side of the corpus callosum. J. Neurosci. 26, 5840-5848.

Kohn, A. D., and Moon, R. T. (2005). Wnt and calcium signaling: betacatenin-independent pathways. Cell Calcium 38, 439-446.

Kuhl, M., Sheldahl, L. C., Malbon, C. C., and Moon, R. T. (2000). $\mathrm{Ca}(2+) /$ calmodulin-dependent protein kinase II is stimulated by Wnt and Frizzled homologs and promotes ventral cell fates in Xenopus. J. Biol. Chem. 275, 12701-12711.

Lau, P. M., Zucker, R. S., and Bentley, D. (1999). Induction of filopodia by direct local elevation of intracellular calcium ion concentration. J. Cell Biol. 145, 1265-1275.

Li, L., Hutchins, B. I., and Kalil, K. (2009). Wnt5a induces simultaneous cortical axon outgrowth and repulsive axon guidance through distinct signaling mechanisms. J. Neurosci. 29, 5873-5883.

Li, Y., Jia, Y. C., Cui, K., Li, N., Zheng, Z. Y., Wang, Y. Z., and Yuan, X. B. (2005). Essential role of TRPC channels in the guidance of nerve growth cones by brain-derived neurotrophic factor. Nature 434, 894-898. 
Lindwall, C., Fothergill, T., and Richards, L. J. (2007). Commissure formation in the mammalian forebrain. Curr. Opin. Neurobiol. 17, 3-14.

Litersky, J. M., Johnson, G. V., Jakes, R., Goedert, M., Lee, M., and Seubert, P. (1996). Tau protein is phosphorylated by cyclic AMP-dependent protein kinase and calcium/calmodulindependent protein kinase II within its microtubule-binding domains at Ser-262 and Ser-356. Biochem. J. 316(Pt 2), 655-660.

Liu, Y., Shi, J., Lu, C. C., Wang, Z. B., Lyuksyutova, A. I., Song, X. J., and Zou, Y. (2005). Ryk-mediated Wnt repulsion regulates posteriordirected growth of corticospinal tract. Nat. Neurosci. 8, 1151-1159.

Livesey, F. J., and Hunt, S. P. (1997). Netrin and netrin receptor expression in the embryonic mammalian nervous system suggests roles in retinal, striatal, nigral, and cerebellar development. Mol. Cell. Neurosci. 8, 417-429.

Lowery, L. A., and Van Vactor, D. (2009). The trip of the tip: understanding the growth cone machinery. Nat. Rev. Mol. Cell Biol. 10, 332-343.

Lucas, F. R., and Salinas, P. C. (1997). WNT-7a induces axonal remodeling and increases synapsin I levels in cerebellar neurons. Dev. Biol. 192, $31-44$.

Luo, L. (2002). Actin cytoskeleton regulation in neuronal morphogenesis and structural plasticity. Annu. Rev. Cell Dev. Biol. 18, 601-635.

Luo, L., and O'Leary, D. D. (2005). Axon retraction and degeneration in development and disease. Annu. Rev. Neurosci. 28, 127-156.

Manitt, C., and Kennedy, T. E. (2002). Where the rubber meets the road: netrin expression and function in developing and adult nervous systems. Prog. Brain Res. 137, 425-442.

McLaughlin, T., and O’Leary, D. D. (2005). Molecular gradients and development of retinotopic maps. Annu. Rev. Neurosci. 28, 327-355.

Metin, C., Deleglise, D., Serafini, T., Kennedy, T. E., and Tessier-Lavigne, M. (1997). A role for netrin-1 in the guidance of cortical efferents. Development 124, 5063-5074.

Ming, G. L., Wong, S. T., Henley, J., Yuan, X. B., Song, H. J., Spitzer, N. C., and Poo, M. M. (2002). Adaptation in the chemotactic guidance of nerve growth cones. Nature 417, 411-418.

Morris, R. L., and Hollenbeck, P. J. (1993). The regulation of bidirectional mitochondrial transport is coordinated with axonal outgrowth. J. Cell Sci. 104(Pt 3), 917-927.
Nakagawa, H., Koyama, K., Murata, Y., Morito, M., Akiyama, T., and Nakamura, Y. (2000). EB3, a novel member of the EB1 family preferentially expressed in the central nervous system, binds to a CNS-specific APC homologue. Oncogene 19, 210-216.

Neal, A. P., Molina-Campos, E., Marrero-Rosado, B., Bradford, A. B., Fox, S. M., Kovalova, N., and Hannon, H. E. (2010). CaMKK-CaMKI signaling pathways differentially control axon and dendrite elongation in cortical neurons. J. Neurosci. 30, 2807-2809.

Nicol, X., Hong, K. P., and Spitzer, N. C. (2011). Spatial and temporal second messenger codes for growth cone turning. Proc. Natl. Acad. Sci. U.S.A. 108, 13776-13781.

Niquille, M., Garel, S., Mann, F., Hornung, J. P., Otsmane, B., Chevalley, S., Parras, C., Guillemot, F., Gaspar, P., Yanagawa, Y., and Lebrand, C. (2009). Transient neuronal populations are required to guide callosal axons: a role for semaphorin 3C. PLoS Biol. 7, e1000230. doi:10.1371/journal.pbio.1000230

Norris, C. R., and Kalil, K. (1992). Development of callosal connections in the sensorimotor cortex of the hamster. J. Comp. Neurol. 326, 121-132.

O'Leary, D. D., Bicknese, A. R., De Carlos, J. A., Heffner, C. D., Koester, S. E., Kutka, L. J., and Terashima, T. (1990). Target selection by cortical axons: alternative mechanisms to establish axonal connections in the developing brain. Cold Spring Harb. Symp. Quant. Biol. 55, 453-468.

Ooashi, N., Futatsugi, A., Yoshihara, F., Mikoshiba, K., and Kamiguchi, H. (2005). Cell adhesion molecules regulate $\mathrm{Ca} 2+-$-mediated steering of growth cones via cyclic AMP and ryanodine receptor type 3 . J. Cell Biol. 170, 1159-1167.

Piper, M., Plachez, C., Zalucki, O., Fothergill, T., Goudreau, G., Erzurumlu, R., Gu, C., and Richards, L. J. (2009). Neuropilin 1-Sema signaling regulates crossing of cingulate pioneering axons during development of the corpus callosum. Cereb. Cortex 19(Suppl. 1), i11-i21.

Polleux, F., Giger, R. J., Ginty, D. D., Kolodkin, A. L., and Ghosh, A. (1998). Patterning of cortical efferent projections by semaphorinneuropilin interactions. Science 282, 1904-1906.

Polleux, F., Morrow, T., and Ghosh, A. (2000). Semaphorin $3 \mathrm{~A}$ is a chemoattractant for cortical apical dendrites. Nature 404, 567-573.
Purro, S. A., Ciani, L., Hoyos-Flight, M., Stamatakou, E., Siomou, E., and Salinas, P. C. (2008). Wnt regulates axon behavior through changes in microtubule growth directionality: a new role for adenomatous polyposis coli. J. Neurosci. 28, 8644-8654.

Qin, S., Yu, L., Gao, Y., Zhou, R., and Zhang, C. (2007). Characterization of the receptors for axon guidance factor netrin- 4 and identification of the binding domains. Mol. Cell. Neurosci. 34, 243-250.

Reh, T., and Kalil, K. (1981). Development of the pyramidal tract in the hamster. I. A light microscopic study. J. Comp. Neurol. 200, 55-67.

Ren, T., Anderson, A., Shen, W. B., Huang, H., Plachez, C., Zhang, J., Mori, S., Kinsman, S. L., and Richards, L. J. (2006). Imaging, anatomical, and molecular analysis of callosal formation in the developing human fetal brain. Anat. Rec. A Discov. Mol. Cell. Evol. Biol. 288, 191-204.

Richards, L. J., Koester, S. E., Tuttle, R., and O'Leary, D. D. (1997). Directed growth of early cortical axons is influenced by a chemoattractant released from an intermediate target. J. Neurosci. 17, 2445-2458.

Rodriguez, J., Esteve, P., Weinl, C., Ruiz, J. M., Fermin, Y., Trousse, F., Dwivedy, A., Holt, C., and Bovolenta, P. (2005). SFRP1 regulates the growth of retinal ganglion cell axons through the $\mathrm{Fz} 2$ receptor. Nat. Neurosci. 8, 1301-1309.

Ruthel, G., and Banker, G. (1999). Role of moving growth cone-like "wave" structures in the outgrowth of cultured hippocampal axons and dendrites. J. Neurobiol. 39, 97-106.

Ruthel, G., and Hollenbeck, P. J. (2000). Growth cones are not required for initial establishment of polarity or differential axon branch growth in cultured hippocampal neurons. $J$. Neurosci. 20, 2266-2274.

Ruthel, G., and Hollenbeck, P. J. (2003). Response of mitochondrial traffic to axon determination and differential branch growth. J. Neurosci. 23, 8618-8624.

Salinas, P. C. (2007). Modulation of the microtubule cytoskeleton: a role for a divergent canonical Wnt pathway. Trends Cell Biol. 17, 333-342.

Salinas, P. C., and Zou, Y. (2008). Wnt signaling in neural circuit assembly. Annu. Rev. Neurosci. 31, 339-358.

Schaefer, A. W., Kabir, N., and Forscher, P. (2002). Filopodia and actin arcs guide the assembly and transport of two populations of microtubules with unique dynamic parameters in neuronal growth cones. J. Cell Biol. $158,139-152$.
Schaefer, A. W., Schoonderwoert, V. T., Ji, L., Mederios, N., Danuser, G., and Forscher, P. (2008). Coordination of actin filament and microtubule dynamics during neurite outgrowth. Dev. Cell 15, 146-162.

Serafini, T., Colamarino, S. A., Leonardo, E. D., Wang, H., Beddington, R., Skarnes, W. C., and Tessier-Lavigne, M. (1996). Netrin-1 is required for commissural axon guidance in the developing vertebrate nervous system. Cell 87 1001-1014.

Shelly, M., Lim, B. K., Cancedda, L., Heilshorn, S. C., Gao, H., and Poo, M. M. (2010). Local and long-range reciprocal regulation of cAMP and cGMP in axon/dendrite formation. Science 327, 547-552.

Sheth, A. N., McKee, M. L., and Bhide, P. G. (1998). The sequence of formation and development of corticostriate connections in mice. Dev. Neurosci. 20, 98-112.

Shim, S., Goh, E. L., Ge, S., Sailor, K., Yuan, J. P., Roderick, H. L., Bootman, M. D., Worley, P. F., Song, H., and Ming, G. L. (2005). XTRPC1dependent chemotropic guidance of neuronal growth cones. Nat. Neurosci. 8, 730-735.

Shimogori, T., VanSant, J., Paik, E., and Grove, E. A. (2004). Members of the Wnt, Fz, and Frp gene families expressed in postnatal mouse cerebral cortex. J. Comp. Neurol. 473, 496-510.

Shu, T., and Richards, L. J. (2001). Cortical axon guidance by the glial wedge during the development of the corpus callosum. J. Neurosci. 21, 2749-2758

Shu, T., Sundaresan, V., McCarthy, M. M., and Richards, L. J. (2003). Slit2 guides both precrossing and postcrossing callosal axons at the midline in vivo. J. Neurosci. 23, 8176-8184.

Singh, K. K., and Miller, F. D. (2005). Activity regulates positive and negative neurotrophin-derived signals to determine axon competition. $\mathrm{Neu}$ ron 45, 837-845.

Singh, K. K., Park, K. J., Hong, E. J., Kramer, B. M., Greenberg, M. E., Kaplan, D. R., and Miller, F. D. (2008). Developmental axon pruning mediated by BDNF-p75NTR-dependent axon degeneration. Nat. Neurosci. 11, 649-658.

Sironi, J. J., Yen, S. H., Gondal, J. A., Wu, Q., Grundke-Iqbal, I., and Iqbal, K. (1998). Ser-262 in human recombinant tau protein is a markedly more favorable site for phosphorylation by CaMKII than PKA or PhK. FEBS Lett. 436, 471-475. 
Kalil et al.

Cortical axon outgrowth, guidance, and branching

Spitzer, N. C. (2006). Electrical activity in early neuronal development. Nature 444, 707-712.

Stepanova, T., Slemmer, J., Hoogenraad, C. C., Lansbergen, G., Dortland, B., De Zeeuw, C. I., Grosveld, F., van Cappellen, G., Akhmanova, A., and Galjart, N. (2003). Visualization of microtubule growth in cultured neurons via the use of EB3GFP (end-binding protein 3-green fluorescent protein). J. Neurosci. 23, 2655-2664.

Takemoto, M., Hattori, Y., Zhao, H., Sato, H., Tamada, A., Sasaki, S., Nakajima, K., and Yamamoto, N. (2011). Laminar and areal expression of unc $5 \mathrm{~d}$ and its role in cortical cell survival. Cereb. Cortex 21, 1925-1934.

Tang, F., Dent, E. W., and Kalil, K. (2003). Spontaneous calcium transients in developing cortical neurons regulate axon outgrowth. J. Neurosci. 23, 927-936.

Tang, F., and Kalil, K. (2005). Netrin-1 induces axon branching in developing cortical neurons by frequencydependent calcium signaling pathways. J. Neurosci. 25, 6702-6715.
Tomida, T., Hirose, K., Takizawa, A., Shibasaki, F., and Iino, M. (2003). NFAT functions as a working memory of $\mathrm{Ca} 2+$ signals in decoding Ca2+ oscillation. EMBO J. 22, 3825-3832.

Uesaka, N., Ruthazer, E. S., and Yamamoto, N. (2006). The role of neural activity in cortical axon branching. Neuroscientist 12, 102-106.

van Ooyen, A. (2011). Using theoretical models to analyse neural development. Nat. Rev. Neurosci. 12, 311-326.

Wang, C. L., Zhang, L., Zhou, Y., Zhou, J., Yang, X. J., Duan, S. M., Xiong, Z. Q., and Ding, Y. Q. (2007). Activitydependent development of callosal projections in the somatosensory cortex. J. Neurosci. 27, 11334-11342.

Wang, G. X., and Poo, M. M. (2005). Requirement of TRPC channels in netrin-1-induced chemotropic turning of nerve growth cones. Nature 434, 898-904.

Wang, Y., Thekdi, N., Smallwood, P. M., Macke, J. P., and Nathans, J. (2002). Frizzled-3 is required for the development of major fiber tracts in the rostral CNS. J. Neurosci. 22, 8563-8573.

Wayman, G. A., Impey, S., Marks, D., Saneyoshi, T., Grant, W. F., Derkach, V., and Soderling, T. R. (2006). Activity-dependent dendritic arborization mediated by CaM-kinase I activation and enhanced CREB-dependent transcription of Wnt-2. Neuron 50, 897-909.

Wayman, G. A., Kaech, S., Grant, W. F., Davare, M., Impey, S., Tokumitsu, H., Nozaki, N., Banker, G., and Soderling, T. R. (2004). Regulation of axonal extension and growth cone motility by calmodulin-dependent protein kinase I. J. Neurosci. 24, 3786-3794.

Wen, Z., and Zheng, J. Q. (2006). Directional guidance of nerve growth cones. Curr. Opin. Neurobiol. 16, 52-58.

Yoshikawa, S., McKinnon, R. D., Kokel, M., and Thomas, J. B. (2003). Wnt-mediated axon guidance via the Drosophila Derailed receptor. Nature 422, 583-588.

Zheng, J. Q., and Poo, M. M. (2007). Calcium signaling in neuronal motility. Annu. Rev. Cell Dev. Biol. 23, 375-404.

Zou, Y., and Lyuksyutova, A. I. (2007). Morphogens as conserved axon guidance cues. Curr. Opin. Neurobiol. 17, 22-28.

Conflict of Interest Statement: The authors declare that the research was conducted in the absence of any commercial or financial relationships that could be construed as a potential conflict of interest.

Received: 20 June 2011; accepted: 08 September 2011; published online: 28 September 2011.

Citation: Kalil K, Li L and Hutchins BI (2011) Signaling mechanisms in cortical axon growth, guidance, and branching. Front. Neuroanat. 5:62. doi: 10.3389/fnana.2011.00062

Copyright (C) 2011 Kalil, Li and Hutchins. This is an open-access article subject to a non-exclusive license between the authors and Frontiers Media SA, which permits use, distribution and reproduction in other forums, provided the original authors and source are credited and other Frontiers conditions are complied with.

Frontiers in Neuroanatomy

www.frontiersin.org

September 2011 | Volume 5 | Article 62 | 15 